\title{
2D Optical Phased Arrays for Laser Beam Steering Based on 3D Polymer Photonic Integrated Circuits
}

\author{
Adam Raptakis, Lefteris Gounaridis, Madeleine Weigel, Moritz Kleinert, Michalis Georgiopoulos, \\ Elias Mylonas, Panos Groumas, Christos Tsokos, Norbert Keil, Hercules Avramopoulos, and Christos \\ Kouloumentas
}

\begin{abstract}
We propose a novel concept for the implementation of 2-dimensional (2D) optical phased arrays (OPAs) with end-fire waveguides as antenna elements (AEs), and we present its theoretical model and experimental proof. The concept is based on the use of 3-dimensional (3D) photonic integrated circuits (PICs) with multiple waveguiding layers on the PolyBoard platform. In their simplest form, the 3D PICs comprise AEs at different layers, vertical and lateral couplers for the distribution of light among the AEs, and phase shifters for the execution of the 2D beam scanning process. Using the field equivalence principle, we model the radiated field from the single-mode waveguide of the platform at $1550 \mathrm{~nm}$, and we find that the expected beam width is $12.7^{\circ}$. We also investigate the perturbation that is induced into propagating fields inside parallel waveguides in proximity, and we conclude that waveguide spacings down to $6 \mu \mathrm{m}$ can be safely used for development of uniform OPAs in the PolyBoard platform. For OPAs with $6 \mu \mathrm{m}$ pitch and 4 AEs, we find that the maximum steering angle is $14.0^{\circ}$ and the expected angular clearance, wherein the main radiation lobe is higher than any grating lobe by at least 3,6 and $10 \mathrm{~dB}$ is $10.8^{\circ}, 7^{\circ}$ and $2.8^{\circ}$, respectively. Based on our simulations, we design and fabricate single- and 2-layer PICs with $1 \times 4$ and $2 \times 4$ OPAs. The lateral pitch of the OPAs ranges from 10 down to $6 \mu \mathrm{m}$, while the vertical pitch is $7.2 \mu \mathrm{m}$. We experimentally characterize these OPAs and validate the potential of the 2-layer PICs for 2D beam scanning on the azimuthal and elevation plane. The beam profiles and the main scanning parameters such as the maximum steering angle and the relative intensity between the main and the grating lobes are found in excellent agreement with our simulations.
\end{abstract}

Index Terms-Optical phased arrays, optical beam scanning, optical polymers, 3D photonic integration, LIDAR systems.

\section{INTRODUCTION}

$\mathrm{O}$ ptical phased arrays (OPAs) have the possibility to replace the moving mirrors and lenses in the laser beam scanning unit of optical sensing and free-space communication modules, enabling realizations of that unit in a compact, robust and low-cost form [1]-[5]. Typical implementations of OPAs in the form of photonic integrated circuits (PICs) bring together a

Manuscript submitted for review on April 22, 2021. This work was supported by the European project H2020-ICT-3PEAT (Contract no. 780502).

A. Raptakis, L. Gounaridis, M. Georgiopoulos, E. Mylonas, P. Groumas, C. Tsokos, H. Avramopoulos, and Ch. Kouloumentas are with the Photonic Communications Research Laboratory, Institute of Communication and Computer Systems at the National Technical University of Athens, Zografou 15573, e-mail:arap@mail.ntua.gr). set of light outcoupling structures that act as optical antenna elements (AEs), a set of phase shifters that adjust the relative phase between the AEs, and a set of optical couplers that split the laser light between the AEs. The far-field of each OPA is the coherent addition of the fields that correspond to the individual AEs, and provided that the arrangement and the phase tuning of those AEs are suitable, this far-field can have a main radiation lobe pointing in a well-controlled direction.

The grating couplers and the end-fire waveguides at the end facet of the PICs have been hitherto the two most common types of optical AEs in OPA implementations [6]. Grating couplers on the surface of planar PICs deflect the propagating light offplane, and act as light emitting structures. In the simplest case, the grating couplers form linear arrays on the surface of the PICs and facilitate the scanning of a laser beam on a single plane with the help of phase shifters on-chip [7]-[10].

Extension of the use of the grating couplers to support beam scanning on two planes, usually referred to as 2-dimensional (2D) scanning, has been achieved using two different concepts. The first one retains the linear arrangement of the grating couplers, but it relies on the dependence of their deflection angle on the operating wavelength. As a result of this dependence, the selection of the wavelength within the tuning range of a laser source can effectively control the beam direction on the plane that is normal to the axis of the linear array, whereas the phase shifters on-chip can still control the beam direction on the plane parallel to that axis. Based on this concept, 2D beam scanning has been demonstrated in the silicon-on-insulator (SOI) and the silicon-nitride photonic platforms with linear arrays of up to 512 AEs using tunable laser sources either on- or off-chip [3],[11]-[17]. The second concept is more straightforward. The grating couplers in that case form 2D (plane) arrays on the surface of the PICs and facilitate in a direct way the $2 \mathrm{D}$ scanning without use of a wavelength tuning mechanism. However, strict requirements regarding the integration density and the maximum length of the grating couplers within the plane arrays are present.

M. Weigel, M. Kleinert and N. Keil are with Fraunhofer Institute for Telecommunications, HHI, Berlin 1058, Germany, (madeleine.weigel@hhi.fraunhofer.de).

P. Groumas and Ch. Kouloumentas are also with Optagon Photonics, Agia Paraskevi 15341, Athens, Greece (christos.kouloumentas@optagonphotonics.eu). 
Leveraging again the high integration density of the SOI platform, these requirements can be partially met, leading to designs of nano-grating couplers with length below $4 \mu \mathrm{m}$, and demonstrations of functional 2D OPAs with number of AEs up to $8 \times 8$ [18]-[22].

The end-fire waveguides represent an alternative type of optical AEs for OPAs. The propagating light finds its way out of the waveguides at the end-facet of the PIC, and is emitted into the air. Compared to the grating couplers, the end-fire waveguides have significant advantages when used in linear arrays for scanning on a single plane: their radiation efficiency is unity, their radiation pattern is smooth and is fully defined by the profile of the waveguided mode, and their size as AEs is the smallest possible one, enabling OPA realizations with large number of AEs and small inter-element spacing (pitch). Using end-fire waveguides, an OPA with 256 AEs for $1550 \mathrm{~nm}$ operation and an OPA with 64 AEs for the blue part of the visible spectrum have been demonstrated in silicon-nitride platforms [23],[24], while OPAs with half-wavelength pitch at $1550 \mathrm{~nm}$ have been developed in SOI [25],[26].

The main drawback of the end-fire waveguides on the other hand is the fact that they cannot form 2D arrays in planar PICs to support the $2 \mathrm{D}$ scanning, which is required by most applications. To overcome this drawback, efforts have been made to develop 3D silica (glass) structures with waveguides at multiple layers that can facilitate 2D OPAs with end-fire waveguides [27],[28]. In one of the most interesting efforts, a $4 \times 4$ OPA was developed [29], using an ultra-fast laser inscription method to inscribe the waveguiding paths in the bulk of the silica structure [30]. Despite the innovation of this work, there are two critical drawbacks of the silica as material system for the implementation of such a concept. The first one is the need for hybrid integration of the silica structure with a PIC that can host the optical couplers and the phase shifters of the OPA. The second one is the weak mode confinement in the silica waveguides that leads to strong optical coupling when the waveguides get in proximity. As a result, the OPA in [29] was designed with a large vertical and lateral pitch of $18 \mu \mathrm{m}$. This led in turn to support a maximum steering angle of only $4.93^{\circ}$, defined as the angular spacing between the main lobe and the grating lobes in the radiation pattern of the OPA.

Among other photonic platforms that can be possibly used for the development of 2D arrays of end-fire waveguides, PolyBoard is maybe the most promising candidate. PolyBoard is a general-purpose polymer platform that supports the monolithic integration of various structures and can act as motherboard for the hybrid integration of heterogeneous elements [31]. The fabrication of PolyBoard PICs involves simple steps, which can be repeated to develop 3D PICs with multiple waveguiding layers and with vertical couplers for light transition between those layers [32]. Such 3D PICs were originally proposed as a solution for coupling to multi-core fibers [33], and later on for the realization of large-scale switching circuits without waveguide crossings [34].

In the present work, we propose for the first time, and we theoretically investigate and experimentally demonstrate the use of 3D PolyBoard PICs with multiple waveguiding layers as
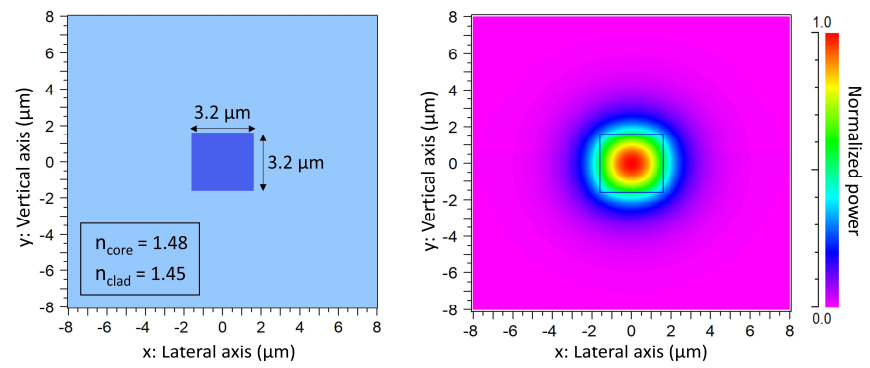

Fig. 1. Cross-section of the single-mode waveguide of the PolyBoard platform at $1550 \mathrm{~nm}$ and intensity profile of the corresponding waveguided mode.

a practical solution for the realization of 2D OPAs with end-fire waveguides. We start from the modelling of the PolyBoard waveguide as an optical antenna at $1550 \mathrm{~nm}$, and find that the full-width at half maximum (FWHM) of its beam is $12.7^{\circ}$. We continue with the modelling of the respective 1D and 2D OPAs, and investigate the beam profile, the maximum steering angle and the relative intensity between the main and the grating lobes of the radiation pattern for different values of the AEs and the pitch. For the value of $6 \mu \mathrm{m}$ in specific, which is identified via beam propagation method (BPM) simulations as a safe pitch limit for the operation of uniform OPAs, we find that the maximum steering angle is $14.0^{\circ}$ and the angular clearance, wherein the intensity of the main lobe is higher than any grating lobe by 3,6 and $10 \mathrm{~dB}$ is $10.8^{\circ}, 7.6^{\circ}$ and $2.8^{\circ}$, respectively. Based on our modelling results, we fabricate a set of $1 \times 4$ and $2 \times 4$ OPAs in single- and 2-layer PolyBoard PICs with all required lateral and vertical couplers and phase shifters on-chip. The lateral pitch in these OPAs varies from 6 to $10 \mu \mathrm{m}$, while the vertical pitch in the subset of the $2 \times 4$ OPAs is $7.2 \mu \mathrm{m}$. Experimental testing of the OPAs validates their possibility for $1 \mathrm{D}$ and $2 \mathrm{D}$ beam scanning, and shows that their radiation parameters are in agreement with the simulation results.

The remainder of the manuscript is organized as follows: In section II, we present the PolyBoard platform and describe its possibility for development of 3D PICs with multiple waveguiding layers and for light transition between those layers via vertical couplers. We describe next how this possibility enables the development of 2D OPAs with end-fire antennas. In section III, we introduce the standard single-mode waveguide of the PolyBoard platform as an elementary optical antenna, we calculate its far-field using the field equivalence principle and the theory of aperture antennas [35], and we extend our modelling to the case of $1 \mathrm{D}$ and $2 \mathrm{D}$ OPAs. In parallel we investigate via BPM simulations the optical crosstalk between the PolyBoard waveguides when parallel and in proximity, and we extract a cut-off pitch for uniform OPAs. In section IV, we present the design and fabrication of PolyBoard PICs with $1 \mathrm{D}$ and 2D OPAs. In section $\mathrm{V}$ we describe the experimental setup for the characterization of those OPAs, and present in detail the corresponding characterization results. Finally, in section IV, we outline our next steps, and conclude.

\section{3D POLYBOARD PLATFORM AND CONCEPT OF 2D OPTICAL PHASED ARRAYS}

PolyBoard is a single-mode photonic platform based on optical polymers $\left(\mathrm{n}_{\text {core }}=1.48, \mathrm{n}_{\text {clad }}=1.45\right)$ that offers low propagation 


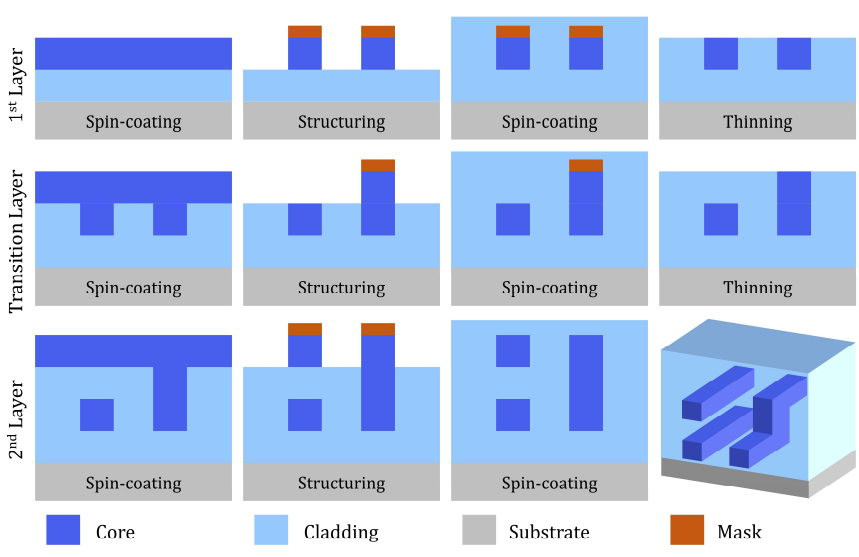

Fig. 2. Process flow with recurring steps for fabrication of 3D PolyBoard PICs with multiple waveguiding layers and vertical MMI couplers for transition of the propagating light between adjacent layers. The example corresponds to a PIC with two waveguiding layers and one vertical MMI coupler.

loss at $1550 \mathrm{~nm}(0.7 \mathrm{~dB} / \mathrm{cm})$ and possibility for fabrication of multi-functional PICs [36]-[38]. This possibility relies on the monolithic integration of elements such as multi-mode interference (MMI) couplers, Mach-Zehnder interferometers, arrayed waveguide gratings, attenuators, optical hybrids and thermal phase shifters, on the hybrid integration of indium phosphide elements such as gain sections, modulators and photodiodes, and on the assembly of thin films and micro-optic elements inside slots and grooves on the surface of the polymer platform [31], [36]. Fig. 1 shows the $3.2 \mu \mathrm{m} \times 3.2 \mu \mathrm{m}$ crosssection of the single-mode waveguide and the mode profile at $1550 \mathrm{~nm}$. Due to the symmetry of the cross-section, the mode is hybrid with a transverse electric (TE) and a transverse magnetic (TM) component. The presence and strength of those components in the propagating field depend on the excitation conditions of the waveguide.

The fabrication of the standard PolyBoard PICs with a single waveguiding layer is based on the use of two polymer resins (waveguide and cladding resin) and successive layer deposition steps. The steps involve the spin-coating of the cladding resin on a silicon $(\mathrm{Si})$ substrate, the spin-coating of the waveguide resin, the structuring of the waveguiding layer using ultra-violet (UV)-lithography and reactive ion etching (RIE), and the spincoating of a second layer of cladding resin to form the top cladding layer. This process can be repeated many times with different mask sets resulting in PolyBoard PICs with multiple waveguiding layers [33]. Within that 3D structure, each layer can be independently formed in a way that retains its potential to support the full set of functionalities offered by PolyBoard technology. Furthermore, the flexibility to use intermediate deposition steps (as shown in the process flow of Fig. 2) enables the structuring of vertical MMI couplers that can couple the light between adjacent layers [32], [34], [39]. Leveraging this 3D integration technology, it becomes possible to develop PolyBoard PICs with lateral MMI couplers, vertical MMI couplers and thermal phase shifters that receive an optical input and distribute this input among a number of output waveguides with precise phase control. Since the waveguides can run at different layers, it is possible to develop a $2 \mathrm{D}$ array of end-fire waveguides at the end-facet of a 3D PolyBoard PIC, enabling

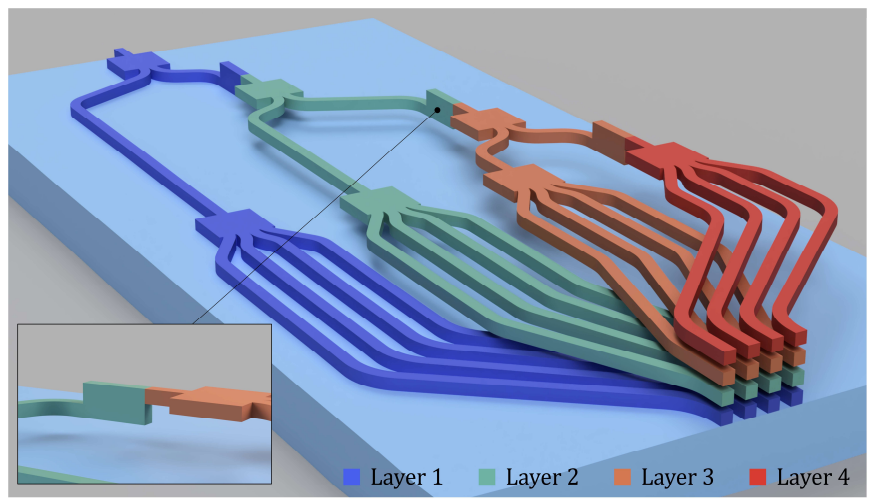

Fig. 3. Concept of 2D OPAs based on PolyBoard PICs with multiple waveguiding layers and vertical MMIs for light coupling between adjacent layers. End-fire single-mode waveguides serve as optical antenna elements at the end-facet of the PolyBoard PIC (cladding material omitted for clarity). A $4 \times 4$ OPA is shown as example. Inset: Close view of a vertical MMI coupler followed bv a lateral one.

in this way the scanning of an optical beam both on the azimuthal and the elevation plane. The pitch of the array in the horizontal direction is determined by the pitch of the end-fire waveguides in the same waveguiding layer, whereas the pitch in the vertical direction by the spacing between the waveguiding layers in the $3 \mathrm{D}$ structure of the PIC. Fig. 3 illustrates the concept of the 2D OPAs based on a 3D PolyBoard PIC taking as example the case of a $4 \times 4$ OPA.

\section{MODELLING AND SIMULATION RESULTS}

In this section, the investigation of the radiation performance of the OPAs in the PolyBoard platform starts from the modelling of the end-fire waveguide as the basic AE. In the next step, the radiation of each OPA in the far-field derives from the combination of the radiation of this $\mathrm{AE}$ with the array factor (AF) that corresponds to the specific OPA [35].

\section{A. Far-field of the end-fire waveguide in PolyBoard PICs}

An end-fire PolyBoard waveguide constitutes a rectangular aperture antenna. The field radiated to the air at the end-facet of the waveguide can be calculated at every point of the hemisphere outside the PolyBoard PIC using the Field Equivalence Principle [35]. Its use involves four steps. In the first one, an imaginary surface that encloses the actual radiation source is defined. In the second step, the actual radiation source is replaced by fictitious sources that reside on the defined surface and yield the same field as the actual source within the volume of interest, which is in fact the volume outside the surface. In the third step, these equivalent sources are calculated using the boundary conditions on the imaginary surface. In order to make this calculation, one has to use the information about the actual value of the electromagnetic field on the surface, and to additionally assume that the corresponding field in the volume that is enclosed by the surface is zero. Finally, in the last step, the equivalent sources that have been calculated in the previous step are used for the calculation of the vector potentials and the electromagnetic field in the volume outside the surface. In the case of an end-fire PolyBoard waveguide with the geometry shown in Fig. 4a, it is convenient to consider the plane at the end-facet of the waveguide as an imaginary surface that extends to the infinity and encloses the radiation 

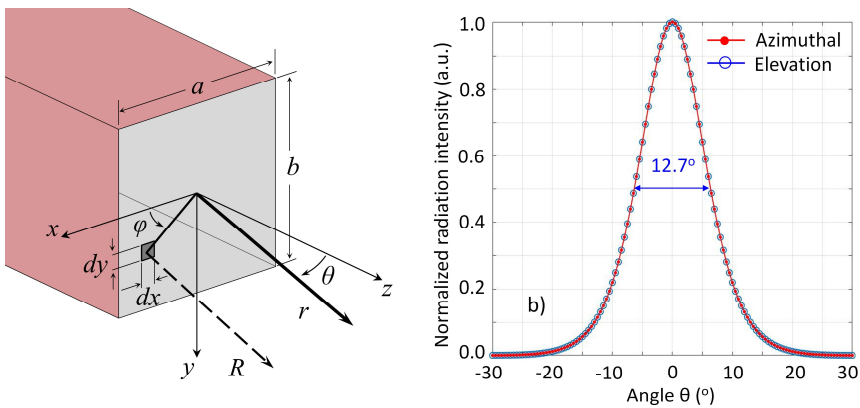

Fig. 4. a) Coordinate system and geometry for the modelling of the end-fire PolyBoard waveguide as an aperture antenna using the Field Equivalence Principle, and b) Normalized radiation intensity of the end-fire PolyBoard waveguide on the azimuthal and the elevation plane.
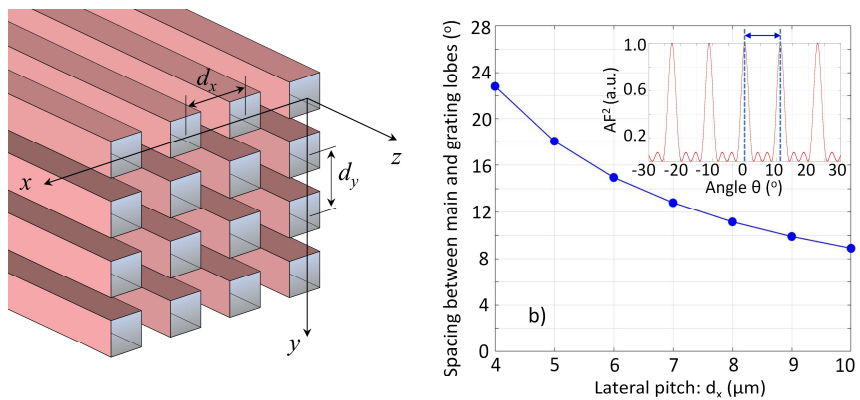

Fig. 5. a) Coordinate system and geometry for the modelling of OPAs with endfire waveguides in PolyBoard PICs, and b) Angular spacing between the main and the grating lobes of the AF (squared) in a linear OPA as a function of the pitch. Results are not PolyBoard specific. Inset: Example AF (squared) corresponding to a linear OPA with 4 AEs, $8 \mu \mathrm{m}$ pitch and $0^{\circ}$ steering angle.

source. With this choice, the information about the mode profile inside the waveguide can be used in order to determine the actual field on the imaginary surface, and eventually to calculate the far-field radiation of the waveguide. The steps and the mathematical operations related to the use of the Field Equivalence Principle in the case of the TE and the TM components of the waveguided mode are presented in detail in the Appendix. The radiation patterns that emerge in the two cases are identical, and have a single lobe. With reference to the spherical coordinate system and the geometry of Fig. 4a, this common pattern has its maximum at $\theta=0$, and is symmetric around the $z$-axis without any dependence on the angle $\varphi$. Fig. $4 \mathrm{~b}$ provides an insight into the radiation intensity $U_{o}\left(r_{o}, \theta, \varphi\right)$ of this pattern at a random radius $r_{o}$ in the far-field. The first curve with red dots in this diagram refers to the azimuthal plane and presents the radiation intensity as a function of $\theta$ for $\varphi=$ $0^{\circ}$ (positive $\theta$-axis) and $\varphi=180^{\circ}$ (negative $\theta$-axis). The second curve with blue empty circles refers to the elevation plane. It has a perfect overlap with the first one and presents the dependence of the radiation intensity on $\theta$ for $\varphi=90^{\circ}$ (positive $\theta$-axis) and $\varphi=270^{\circ}$ (negative $\theta$-axis). It is noted that in reality, the angle $\theta$ takes only positive values. However, in the diagram of Fig. $4 \mathrm{~b}$, we use both the positive and the negative part of the axis to discriminate between the $\theta$ values that correspond to $\varphi=90^{\circ}$ and those that correspond to $\varphi=$ $270^{\circ}$ or between the $\theta$ values that correspond to $\varphi=0$ and those that correspond to $\varphi=180^{\circ}$. On both planes, the FWHM of the radiation intensity is approximately $12.7^{\circ}$. Since the radiation pattern is symmetric around the $z$-axis, the FWHM remains the same for any angles $\varphi$ and $\varphi+180^{\circ}$, and describes unambiguously the directivity of the PolyBoard end-fire waveguide as an optical antenna.

Finally, it is noted that in the analysis so far, the spherical coordinates have been based on the definition of the $\theta$ and $\varphi$ angles, but they can be also based on the azimuth $(\alpha z)$ and elevation $(e l)$ angles instead. The first one is the angle between the $z$-axis and the orthogonal projection onto the $x z$-plane of the vector between the origin and the observation point, and is positive when going from the $z$ - towards the $x$-axis. The second one is the angle between the same vector and its orthogonal projection onto the $x z$-plane, and is positive when going from the $x z$-plane towards the negative $y$-axis.

\section{B. Far-Field of $1 D$ optical phased arrays in PolyBoard PICs}

In PolyBoard PICs with a single waveguiding layer, the endfire waveguides act as a set of identical AEs that form a linear OPA. In the case of uniform OPAs, the pitch is constant, the excitation fields have the same magnitude, and the differential phase $\beta$ in the excitation of each AE compared to its preceding one remains the same for all AEs. With reference to the definition of the axes in Fig. 5a, the array factor (AF) of a uniform OPA is expressed as per the well-known relation [35]:

$$
A F(\theta, \varphi)=\sum_{n=1}^{N} e^{i \cdot(n-1) \cdot\left(k \cdot d_{x} \cdot \sin \theta \cos \varphi+\beta_{x}\right)}
$$

where $N$ the number of antenna elements, $d_{x}$ the pitch of the linear array and $k$ the wavenumber in the free-space. The direction of the main lobe of the AF on the azimuthal plane is controlled by the parameter $\beta$. Since however the pitch of the PolyBoard OPAs is much larger than the half of the wavelength at $1550 \mathrm{~nm}$, grating lobes are also present in the $\mathrm{AF}$, setting limitations on the maximum steering angle and the field-ofview (FOV) that are offered by the OPAs. The inset of Fig. 5b presents as an example the main and the grating lobes of the square of the AF that corresponds to a 4-element linear OPA at $1550 \mathrm{~nm}$ with $8 \mu \mathrm{m}$ pitch and with $0^{\circ}$ steering angle on the azimuthal plane. The main diagram of Fig. $5 \mathrm{~b}$ presents on the other hand the angular spacing between the main and the grating lobes of the square of the AF as a function of the pitch. Although the dependence shown in this diagram is general and holds true for all uniform OPAs, it is of particular value for the design of PolyBoard OPAs, since the latter are based on waveguides that are rather wide $(3.2 \mu \mathrm{m})$ and offer weak mode confinement due to their low refractive index contrast.

Using the radiation intensity $\left(U_{o}\right)$ of the PolyBoard end-fire waveguide and the AF of a linear OPA, the radiation intensity $(U)$ of a single-layer PolyBoard OPA is calculated as follows:

$$
U(r, \theta, \varphi)=U_{o}(r, \theta, \varphi) \cdot[A F(\theta, \varphi)]^{2}
$$

Fig. 6a presents two example cases for the radiation intensity of such an OPA on the azimuthal plane. The first one is shown with red solid line and corresponds to an OPA with 4 AEs, 8 $\mu \mathrm{m}$ pitch and steering direction at $4^{\circ}$. The second one is shown with blue dotted line and corresponds to an OPA with 8 AEs, 6 $\mu \mathrm{m}$ pitch and direction at $-4^{\circ}$. The radiation intensity of the basic AE is also illustrated as an envelope in agreement with the physical meaning of Eq. (2). In both cases, only one grating 

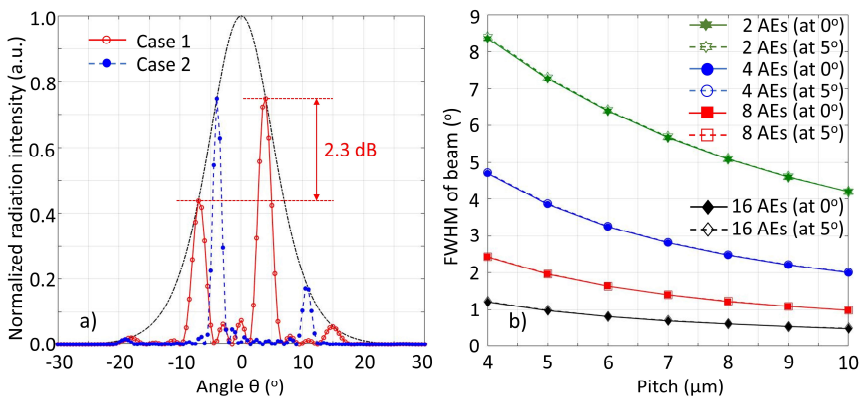

Fig. 6. a) Exemplary radiation intensity of two linear PolyBoard OPAs on the azimuthal plane: The first one (Case 1) has 4 AEs, $8 \mu \mathrm{m}$ pitch and direction at $+4^{\circ}$, and the second one (Case 2) has 8 AEs, $6 \mu \mathrm{m}$ pitch and direction at $-4^{\circ}$. b) FWHM of the main lobe of the radiation intensity as a function of the pitch for different number of AEs and steering angles.
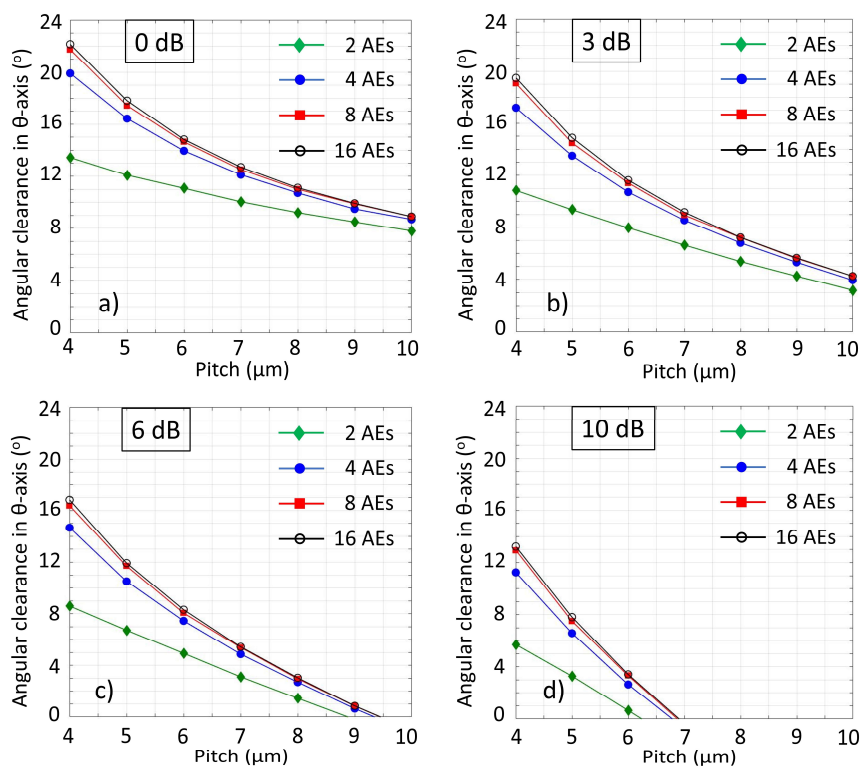

Fig. 7. Radiation intensity of linear PolyBoard OPAs on the azimuthal plane: Angular clearance, wherein the main lobe of the radiation pattern is higher than lobe is clearly observed due to the suppression imposed on all the other ones. The angular spacing between each main lobe and its companion grating lobe is primarily defined by the pitch of the respective OPA. It is noted however that this spacing is slightly smaller than the corresponding spacing in Fig. $5 \mathrm{~b}$ between the lobes of the $\mathrm{AF}^{2}$. This reduction is a result of the multiplication between the $\mathrm{AF}^{2}$ and the radiation intensity of the basic AE, and is present for any pitch or number of AEs. Finally, Fig. $6 \mathrm{~b}$ presents results regarding the beam width in the radiation pattern of a PolyBoard OPA. It reveals the strong dependence of the FWHM of the main lobe on the number of AEs, and the weaker dependence on the waveguide pitch. On the other hand, no dependence on the steering angle can be observed for angles that remain within the range of interest.

Looking more carefully at the main and the grating lobe in the first case of Fig. $6 \mathrm{a}$, we find that their relative intensity ratio is $2.3 \mathrm{~dB}$. If the beam is steered further to the right with $\theta$ larger than $4^{\circ}$, this ratio drops. If on the contrary, the beam is pulled back to the other direction, the ratio increases and gets back to its initial value only when the main lobe is at $-4^{\circ}$. The angular space from $-4^{\circ}$ to $4^{\circ}$ represents in this example the symmetric clearance around $0^{\circ}$, wherein the main lobe is larger than any grating lobe by at least $2.3 \mathrm{~dB}$. Figs. $7 \mathrm{a}$ to $7 \mathrm{~d}$ extend this investigation and present the clearance around $0^{\circ}$, wherein the main lobe of the radiation pattern remains larger than any grating lobe by at least $0,3,6$ or $10 \mathrm{~dB}$, respectively. By default, the $0 \mathrm{~dB}$ clearance shown in Fig. 7a reveals the spacing between the main and the grating lobes for the respective number of AEs and waveguide pitch. On the other hand, the $3 \mathrm{~dB}, 6 \mathrm{~dB}$ and 10 $\mathrm{dB}$ clearance can serve as a practical metric for the assessment of the attainable FOV, depending on the grating lobe suppression requirements of each application. In all cases of Fig. 7, the clearance has strong dependence on the waveguide pitch. Since however it has strong dependence also on the radiation intensity profile of the basic $\mathrm{AE}$, the four diagrams are valid and hold true only in the particular case of PolyBoard OPAs with end-fire waveguides, which is studied in this work.

\section{Far-Field of 2D optical phased arrays in PolyBoard PICs}

In PolyBoards with multiple waveguiding layers, the end-fire waveguides form a plane OPA. The radiation properties presented in the previous paragraph for the azimuthal plane in the case of linear OPA (see Figs. 5-7) can be extended without modification to the elevation plane to describe the radiation pattern of a plane OPA and the possibility for 2D scanning of that pattern on the azimuthal and the elevation plane. Using again the spherical coordinate system and the definition of the axes in Fig. 4a, the AF of a uniform plane OPA is given as:

$$
\begin{aligned}
& A F(\theta, \varphi)= \\
& =\sum_{n=1}^{N} \sum_{m=1}^{M} e^{i \cdot(n-1) \cdot\left(k \cdot d_{x} \cdot \sin \theta \cos \varphi+\beta_{x}\right)} e^{i \cdot(m-1) \cdot\left(k \cdot d_{y} \cdot \sin \theta \sin \varphi+\beta_{y}\right)}
\end{aligned}
$$

where $N$ and $d_{x}$ the number of AEs and the pitch along the xaxis, while $M$ and $d_{y}$ the number of AEs (or layers) and the pitch along the $y$-axis. The total radiation intensity $(U)$ is given again by Eq. (2), using the radiation intensity $\left(U_{o}\right)$ of the endfire waveguide and the AF of the plane OPA from Eq. (3).

\section{Optical crosstalk between the PolyBoard waveguides}

The results in Figs. 6-7 for the linear OPAs in single-layer PolyBoards and the extension of these results to the case of the plane OPAs in multi-layer PolyBoards are based on the assumption that the phase of each $\mathrm{AE}$ can be independently controlled. This holds true, when the pitch of the OPAs is large enough, but it does not when the waveguides are brought in proximity and start getting coupled. Within this context, parts of the diagrams in Fig. $6 \mathrm{~b}$ and Figs. 8a-d may not be of any practical value since they might correspond to waveguide spacings that do not prevent this kind of detrimental coupling. To evaluate the strength of the waveguide coupling as a function of the pitch and define a conventional cutoff pitch as a guideline for the design of OPAs in single- and multi-layer PolyBoards, we take the simplest case of two parallel PolyBoard waveguides with $100 \mu \mathrm{m}$ length (see Fig. 8a), and we simulate the light propagation in those waveguides using the Beam Propagation Method (BPM) [40]-[41]. Both waveguides are excited by their fundamental eigenmode with $\mathrm{TE}$ polarization and peak amplitude normalized to unity. In all simulations for a particular pitch, the phase of the excitation 

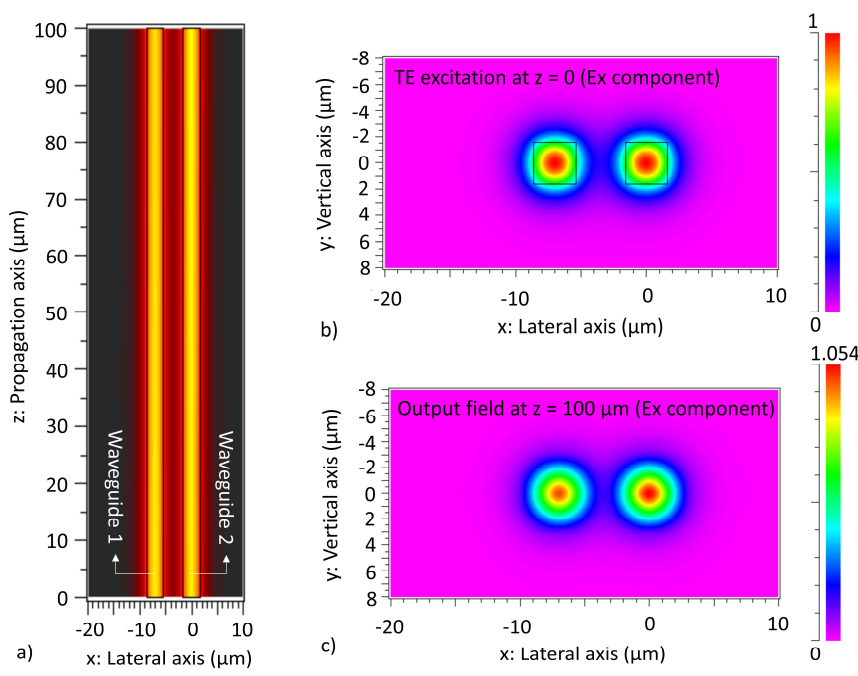

Fig. 8. BPM study for the estimation of the crosstalk between two PolyBoard waveguides: a) BPM pattern for $100 \mu$ m propagation, b) TE excitation of the two waveguides, and c) Output fields with dominant Ex component at $z=100$ $\mu \mathrm{m}$. The three diagrams correspond to $7 \mu \mathrm{m}$ waveguide pitch, $-30^{\circ}$ phase in the excitation of waveguide 1 , and $0^{\circ}$ phase in the excitation of waveguide 2 .
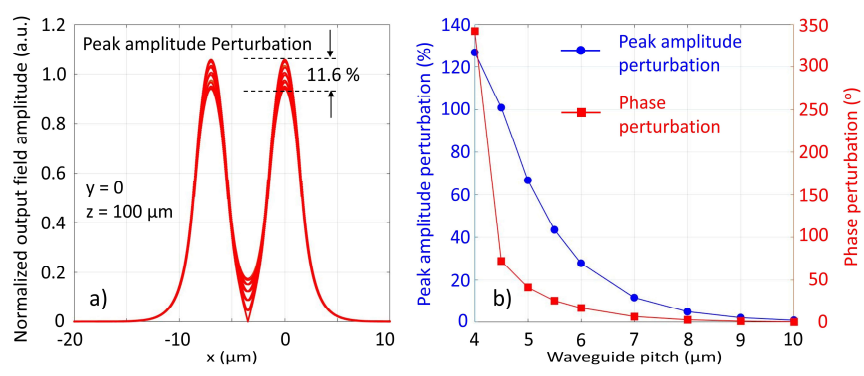

Fig. 9. a) Amplitude perturbation in the output field of waveguide $2(\mathrm{z}=100$ $\mu \mathrm{m})$ due to the phase variation in the excitation of waveguide 1 (see Fig. 9a). The 12 curves correspond to excitation phase from $-180^{\circ}$ to $+150^{\circ}$ with $30^{\circ}$ step. b) Main BPM results: Amplitude and phase perturbation at waveguide 2 output as a function of the pitch. The values are for the center of waveguide 2 ( $x=0$, $\mathrm{y}=0)$.

field in the right-most waveguide (denoted as waveguide 2) is zero, whereas the phase of the excitation field in the left-most waveguide (denoted as waveguide 1) varies from $-180^{\circ}$ to $+180^{\circ}$. Fig. 8 presents as example a case that corresponds to 7 $\mu \mathrm{m}$ pitch and $-30^{\circ}$ phase of the excitation field at the input of waveguide 1. More specifically, Fig. 8a illustrates the beam propagation pattern and reveals in a qualitative way the optical cross-talk between the two waveguides. Fig. $8 \mathrm{~b}$ presents in turn the cross-section of the two waveguides and the distribution of the TE fields that are employed for the excitation of the two waveguides at $z=0$. Finally, Fig. 8c depicts the corresponding distribution of the output fields at $z=100 \mu \mathrm{m}$, and makes evident the asymmetry that is induced between the two waveguides due to the optical crosstalk. The perturbation of the amplitude and the phase of the output field of waveguide 2 with respect to the corresponding amplitude and phase of the output field, when this waveguide is alone, depends both on the pitch and on the phase of the excitation field at the input of waveguide 1 . The level of this perturbation at the center of the cross-section of waveguide 2 is used hereafter as metric for the assessment of the coupling between the two waveguides.

In more detail, Fig. 9a presents as an example the peak amplitude perturbation of the output fields, when the pitch is 7
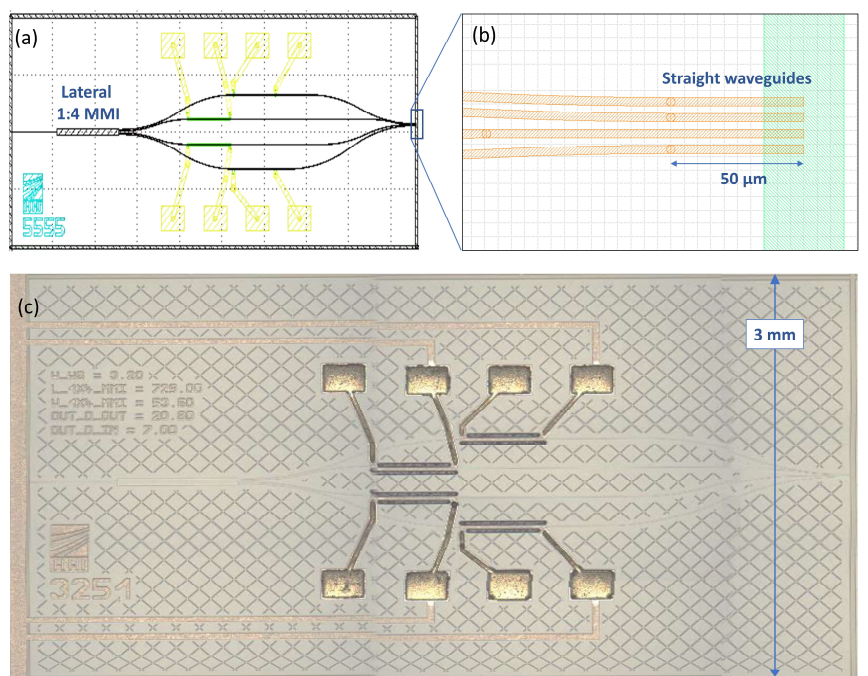

Fig. 10. a) Mask layout for the fabrication of $1 \times 4$ OPAs with $6 \mu \mathrm{m}$ lateral pitch in single-layer PolyBoards, (b) Detail of the layout in the left most section, where the waveguides run in parallel, and c) Micro-photograph of a respective PolyBoard PIC (top-view).
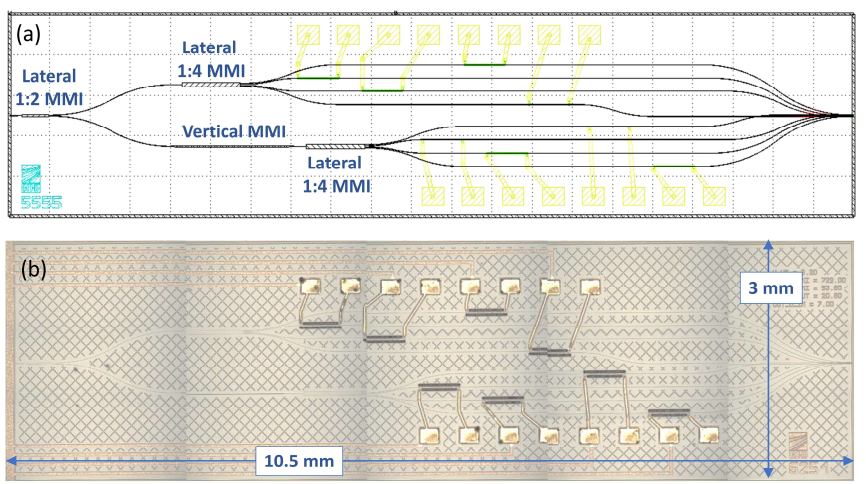

Fig. 11. a) Mask layout for the fabrication of $2 \times 4$ OPAs with $6 \mu \mathrm{m}$ lateral and 7.2 vertical pitch in 2-layer PolyBoards, and (b) Micro-photograph of a respective PolyBoard PIC (top-view).

$\mu \mathrm{m}$ and the excitation phase at the input of waveguide 1 varies from $-180^{\circ}$ to $+150^{\circ}$ with $30^{\circ}$ step (i.e. 12 curves in total). The perturbation is the same for both waveguides with a range of almost $11.6 \%$ of the peak amplitude at the input. The range of the phase perturbation is not shown in this diagram, but it is $6.6^{\circ}$ in absolute terms. Fig. 9b summarizes the ranges of the amplitude and phase perturbation as a function of the waveguide pitch for values between 4 and $10 \mu \mathrm{m}$. As shown, for pitch equal or larger than $8 \mu \mathrm{m}$, the perturbation is negligible, and the waveguides remain practically decoupled. For pitch between 8 and $6 \mu \mathrm{m}$, the perturbations start rising, but remain moderate and manageable, whereas for pitch below 6 $\mu \mathrm{m}$, the rise becomes abrupt. Based on these remarks, we can conclude that the $6 \mu \mathrm{m}$ can be a safe limit for the pitch of uniform OPAs in the PolyBoard platform.

\section{DESIGN AND FABRICATION}

Two types of PolyBoard PICs were developed to provide the experimental proof-of-concept regarding the potential of the PolyBoard platform for laser beam scanning. The first type corresponds to single-layer PICs with linear $1 \times 4$ OPAs. Three versions of these PICs were designed with lateral pitch equal to 
6,8 and $10 \mu \mathrm{m}$, respectively, in order to experimentally investigate the impact of the pitch on the beam parameters and the beam scanning performance. Fig. 10 presents the mask layout and a micro-photograph of the specific version that corresponds to $6 \mu \mathrm{m}$ pitch. On the left side of the circuit, the input light is split into four equal parts by a lateral 1:4 MMI coupler. In the output waveguides of the coupler, heating electrodes with $20 \mathrm{Ohm}$ resistance serve as thermal phase shifters for adjustment of the phase relation between the AEs. The required current at each heating electrode for pi-phase shift is approximately $16-20 \mathrm{~mA}$. Finally, in the left-most part of the circuit, the four waveguides are brought in proximity via carefully designed S-bends, and run in parallel towards the endfacet of the PIC. The length of this very last section, where the waveguides run completely in parallel (see Fig. 10b) is short $(50 \mu \mathrm{m})$ so as to keep the crosstalk between the parallel waveguides as low as possible.

The second type of PolyBoards corresponds to PICs with two waveguiding layers that support the development of $2 \times 4$ OPAs. Three versions of these PICs were designed with lateral pitch equal to 6,8 and $10 \mu \mathrm{m}$, respectively. The vertical pitch was 7.2 $\mu \mathrm{m}$ in all versions. Fig. 11 presents the mask layout and a micro-photograph of the specific version with $6 \mu \mathrm{m}$ lateral pitch. On the left side of the circuit the input signal is split in two parts by a lateral 1:2 MMI coupler. The light at the second output of this coupler is transferred to the upper waveguiding layer by means of a vertical MMI coupler with $1350 \mu \mathrm{m}$ length and $10.4 \mu \mathrm{m}$ height. At both layers, the rest of the optical circuit is practically the same as the circuit of the single layer PICs described above: the light at each layer is split in four equal parts by a lateral 1:4 MMI coupler and the optical phase inside each output waveguide is adjusted by a thermal phase shifter. Finally, the four waveguides are brought in proximity by means of S-bends and run in parallel till the end-facet of the PIC. It is noted that the two linear $1 \times 4$ OPAs at the two layers are laterally aligned to each other so as to form a perfectly rectangular $2 \times 4$ OPA.

The fabrication of the single-layer PICs was based on the standard steps of PolyBoard technology. The fabrication of the 2-layer PICs on the other hand was realized using the sequence of spin-coating, structuring and removal steps of the 3D PolyBoard technology that were outlined in section II. After the end of the wafer processing, all PolyBoard PICs were diced and polished, and they were taken further for experimental testing.

\section{EXPERIMENTAL CHARACTERIZATION}

In this section we present the setup for the experimental characterization of the PolyBoard PICs, and the main results that were obtained as the core outcome of this characterization.

\section{A. Experimental setup}

Fig. 12 depicts the setup for the experimental investigation of the radiation pattern of the $1 \mathrm{D}$ and $2 \mathrm{D}$ OPAs in the developed PolyBoard PICs. A distributed feedback (DFB) laser provides a continuous wave (cw) at $1563 \mathrm{~nm}$ with $-5.0 \mathrm{dBm}$ output power. The light passes through an optical isolator and a polarization controller (PC), and is coupled to each PIC under test from the left-side of the PIC. It further propagates through the optical structures on-chip, is emitted from the end-fire
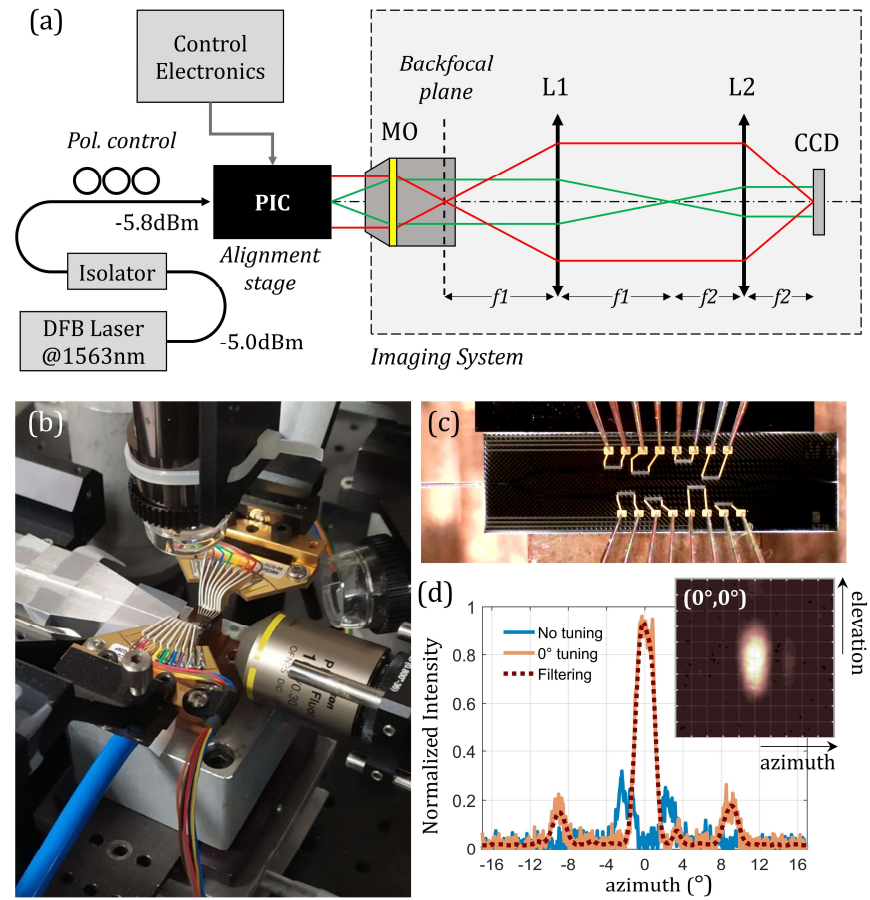

Fig. 12. (a) Layout of experimental setup and Fourier imaging system for the characterization of the far-field of the PolyBoard OPAs. (b) Picture of the setup. (c) Close-up of a PolyBoard PIC with a $2 \times 4$ OPA. (d) Example image from a $2 \times 4$ OPA with $6 \mu \mathrm{m}$ lateral pitch. The beam is centered at $0^{\circ}$ on both planes. The diagram depicts the plots related to the radiation intensity on the azimuthal plane when the phase shifters are off, when the phase shifters are tuned for beam

waveguides on the right-hand side of the PIC, and is collected by a system of lenses that form a Fourier imaging system [42]. In such a system, the far-field is imaged at the back-focal (Fourier) plane of a microscope objective (MO), and is brought back to a sensor using a pair of lenses with an image ratio, which is defined by the focal lengths of the two lenses. The MO in our setup has a numerical aperture (NA) equal to 0.3 . The two lenses L1 and L2 have focal lengths $\mathrm{f} 1$ and $\mathrm{f} 2$ equal to 100 and $50 \mathrm{~mm}$, respectively. These lengths have been carefully selected so that the entire area of the sensor at the right end of the imaging system can be utilized for light detection. This sensor is in fact a $1 / 2$ " charge-coupled device (CCD) near infrared (NIR) camera with $768 \times 494$ pixels and $8.4 \mu \mathrm{m} \times 9.8 \mu \mathrm{m}$ pixel size. With this imaging system, emission angles from the OPAs up to $17^{\circ}$ on the azimuthal plane and $14^{\circ}$ on the elevation plane can be measured with resolution better than a tenth of degree.

The emitted light that passes through the principal axis of the imaging system hits the center of the CCD sensor, and appears at the center of the captured image, corresponding to $0^{\circ}$ beam steering angle both on the azimuthal and the elevation plane. The light that is emitted towards the positive azimuthal angle corresponds to the right part of the image, while the light that is emitted towards the positive elevation angle corresponds to the upper part of the image. Prior to the installation of the imaging system in our setup, a careful characterization was carried out to create a pixel-to-angle mapping for the captured images. For this purpose, an auxiliary laser source with a collimated output beam was mounted on a rotational stage [43]. The rotation axis of the stage was placed exactly at the position, where the OPAs at the end-facet of the PolyBoard PICs were expected to be in 


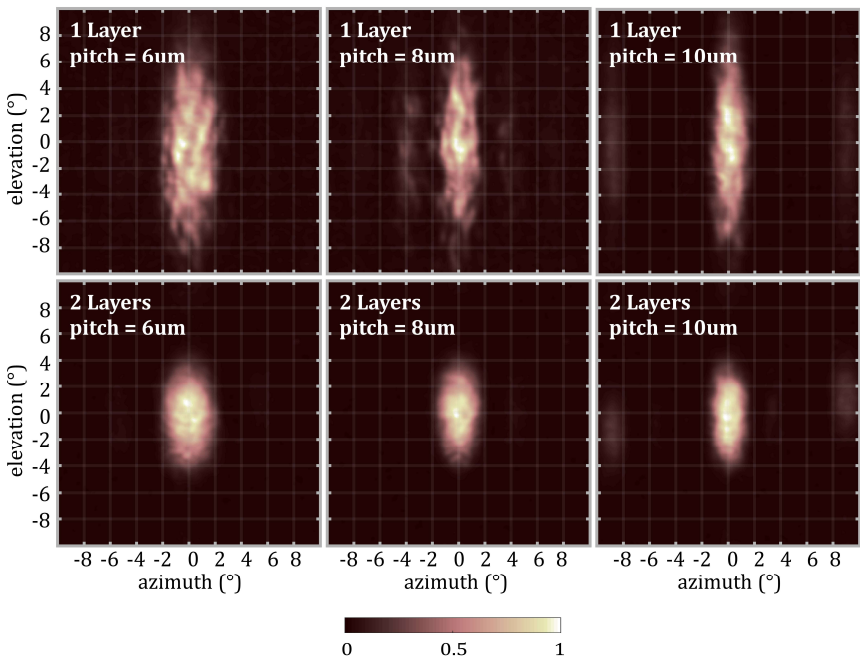

Fig. 13. Beam profile for all the OPAs in the PolyBoard PICs under test: The upper row corresponds to $1 \times 4$ OPAs in single-layer PICs, whereas the lower row to $2 \times 4$ OPAs in 2-layer PICs. The lateral waveguide pitch is 6,8 or $10 \mu \mathrm{m}$. The vertical pitch in the 2-layer OPAs is fixed at $7.2 \mu \mathrm{m}$.
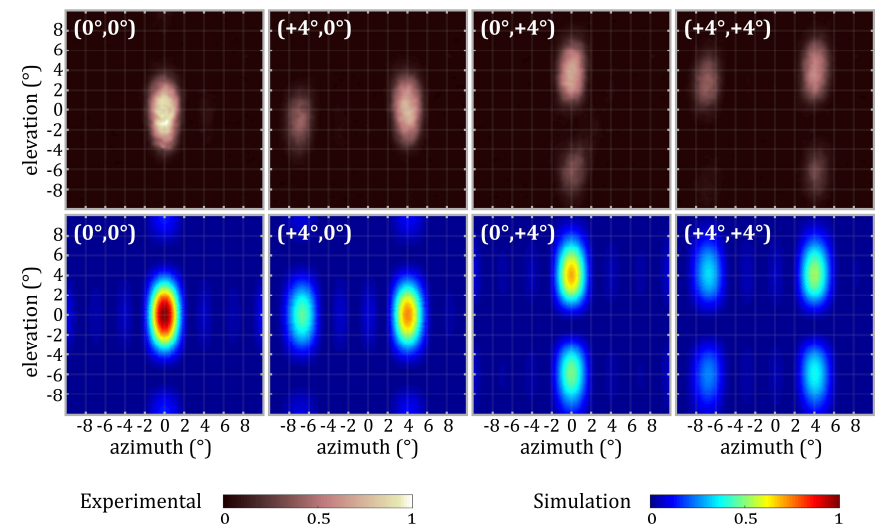

Fig. 14. Comparison between the simulation (upper row) and the experimental (lower row) radiation patterns in the case of the $2 \times 4$ OPA with $8 \mu \mathrm{m}$ lateral pitch in four beam steering scenarios. The intended beam steering angle of the main lobe is displayed in each frame at the top-left: $1^{\text {st }}$ column scenario: $\left(0^{\circ}\right.$, $\left.0^{\circ}\right) .2^{\text {nd }}$ column scenario: $\left(4^{\circ}, 0^{\circ}\right) \cdot 3^{\text {rd }}$ column scenario: $\left(0^{\circ}, 4^{\circ}\right) \cdot 4^{\text {th }}$ column scenario: $\left(4^{\circ}, 4^{\circ}\right)$.

order to emulate the light emission conditions in the actual experiments. It is noted that the beam size of the auxiliary laser source was adequately small to yield a spot size of almost a single dot in our imaging system. Via the rotation of the collimated beam by a known angle, it was thus possible to calibrate the image acquisition process in terms of steering angle and light intensity, and to compensate for the small image distortion effects originating from our lens system.

Light coupling into the PolyBoard PIC under test is accommodated by a 6-axis alignment station. As already mentioned, a polarization controller adjusts the polarization state of the light prior to PIC. Despite the polarization insensitivity of the PolyBoard waveguides, a weak polarization dependence across the integrated circuit is still expected due to the non-perfect polarization insensitivity of the lateral and vertical MMI couplers. The heating electrodes that adjust the phase of the individual AEs of the OPAs are controlled by an 8 -channel current driver. All channels are used for the $2 \times 4$ OPAs, whereas only half of them for the $1 \times 4$ OPAs. Two 16 - pin probe heads with $50 \mu \mathrm{m}$ pitch are additionally used to interface the controller with the chip pads. Finally, a Pythonbased code has been developed for capturing frames from the CCD sensor and processing the images. A Gaussian smoothing filter can be also applied for suppression of the white noise from the sensor without altering the captured pattern. Fig. 12d presents as example the intensity distribution of the captured image at $0^{\circ}$ elevation angle, when the phase shifters are off, when the phase shifters are tuned for $0^{\circ}$ steering on the azimuthal plane, and when the filter is additionally applied. The inset illustrates the corresponding 2D image in the last case.

\section{B. Experimental results}

Fig. 13 presents the profile of the radiated beam for all the fabricated versions of the PolyBoard OPAs. The FWHM of the beam intensity in the case of the single-layer OPAs is measured $3.3^{\circ} \times 12.7^{\circ}, 2.6^{\circ} \times 12.4^{\circ}$ and $2.1^{\circ} \times 12.9^{\circ}$ for lateral pitch equal to $6 \mu \mathrm{m}, 8 \mu \mathrm{m}$ and $10 \mu \mathrm{m}$, respectively. As observed, the width of the beam on the elevation plane approximates the beam width of the single waveguide emitter, as there are no additional waveguides in the vertical direction to act on the array factor. On the azimuthal plane on the other hand, the beam width is becoming narrower, as the pitch is increasing. This dependence has been already described in the modelling section (see Fig. $6 \mathrm{~b}$ ), and the experimental data fit very well to the simulation results. In the case of the 2-layer PolyBoard PICs, the FWHM values are $3.3^{\circ} \times 5.9^{\circ}, 2.6^{\circ} \times 5.7^{\circ}$ and $2.1^{\circ} \times 5.8^{\circ}$ for lateral pitch equal to $6 \mu \mathrm{m}, 8 \mu \mathrm{m}$ and $10 \mu \mathrm{m}$, respectively. As observed, the addition of a second emission layer decreases drastically the beam width in the vertical direction, demonstrating the strong impact of the number of the AEs on the beam divergence. In the lateral direction, the beam is becoming again narrower as the pitch is increasing. It is worth noting that regardless of the number of the waveguiding layers (either one or two), the FWHM in the lateral direction remains the same for the same lateral pitch, indicating the high performance of the PolyBoard PICs and the consistency of the measurements.

The OPAs in all PolyBoard PICs were fabricated with random phase differences between the AEs, resulting in a random radiation pattern when the phase shifters are off (see Fig. 12d). To overcome the randomness of this initial pattern, each PIC has to undergo a 2-step calibration process. In the first step, the required driving current for phase shift equal to $2 \pi$ is identified for each phase shifter (heating electrode). In the second step, appropriate phase shifts are applied on the individual waveguides in order to eliminate the phase differences between the AEs and maximize the radiation intensity at the direction of $0^{\circ}$ on both the azimuthal and the elevation plane. After the execution of these steps, all required information for precise 2D beam scanning is known. In all PolyBoard PICs under test, the required current for $2 \pi$ phase shift was found to be close to $20 \mathrm{~mA}$ for the bottom layer electrodes and close to $16 \mathrm{~mA}$ for their top layer counterparts.

Fig. 14 presents a comparison between experimental and simulation results as an evidence for the potential of the $3 \mathrm{D}$ PolyBoard PICs to support well-controlled beam scanning on the azimuthal and the elevation plane. The experimental images (in the upper row) are from the testing of the $2 \times 4$ OPA with 8 $\mu \mathrm{m}$ lateral pitch, and the simulation results (in the lower row) are from the simulation of the far-field radiation of the same 

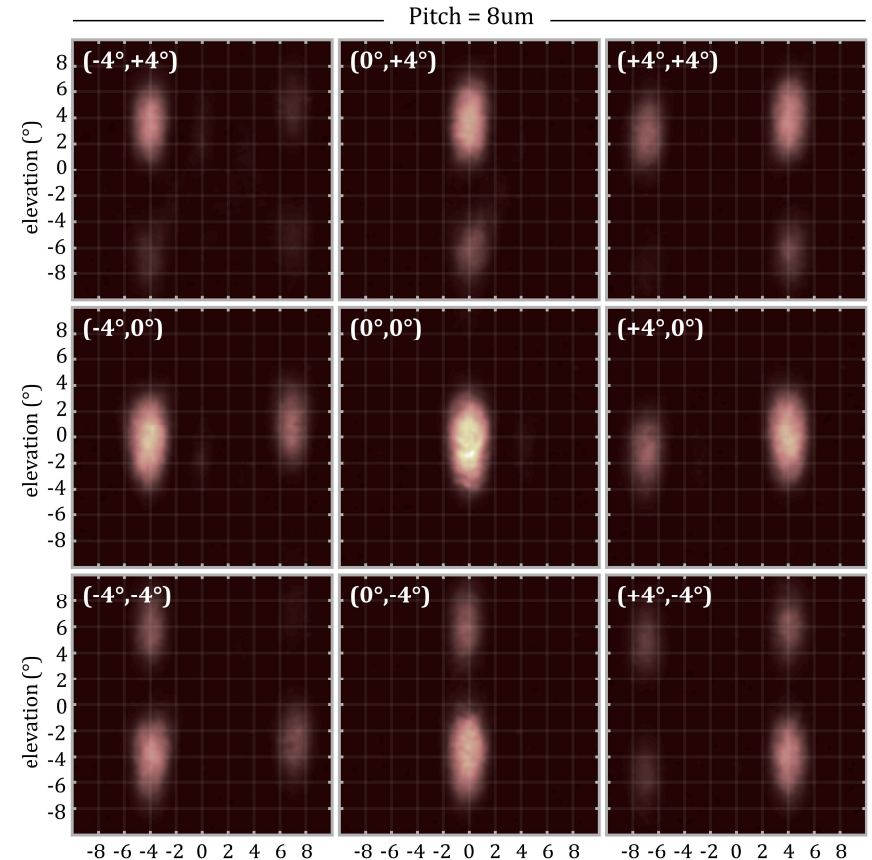

$\begin{array}{lllllllll}-8 & -6 & -4 & -2 & 0 & 2 & 4 & 6 & 8\end{array}$ azimuth $\left({ }^{\circ}\right)$

Pitch $=6$ um
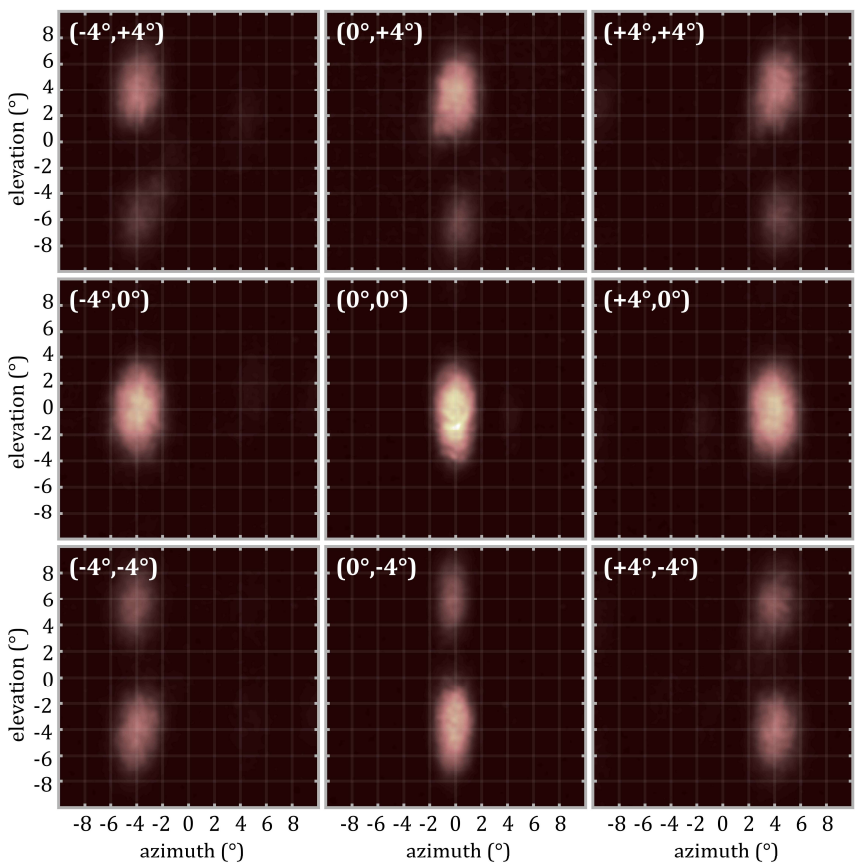

0

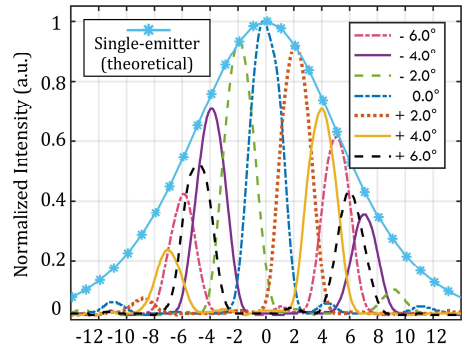

(a)

azimuth $\left({ }^{\circ}\right)$

(b)
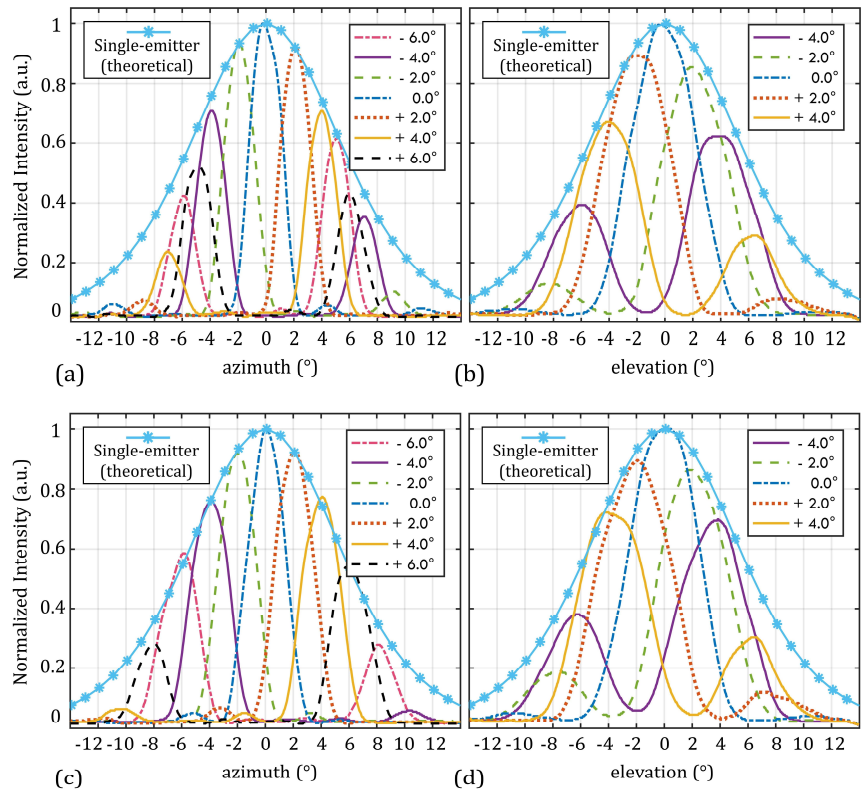

Fig. 16. Analysis of experimental images from two $2 \times 4$ OPAs with 8 and $6 \mu \mathrm{m}$ lateral pitch. Eleven beam steering scenarios are investigated for each OPA corresponding to $0^{\circ}$ elevation angle and azimuthal angle from $-6^{\circ}$ to $+6^{\circ}$ with $2^{\circ}$ step, and to $0^{\circ}$ azimuthal angle and elevation angle from $-4^{\circ}$ to $+4^{\circ}$ with $2^{\circ}$ step: a) $8 \mu \mathrm{m}$ pitch: Intensity distribution on the azimuthal plane for $0^{\circ}$ elevation angle. b) $8 \mu \mathrm{m}$ pitch: Intensity distribution on the elevation plane for $0^{\circ}$ azimuthal angle. c) $6 \mu \mathrm{m}$ pitch: Intensity distribution on the azimuthal plane for $0^{\circ}$ elevation angle. d) $6 \mu \mathrm{m}$ pitch: Intensity distribution on the elevation plane for $0^{\circ}$ azimuthal angle. In all diagrams, the theoretical intensity distribution of the basic emitter (PolyBoard end-fire waveguide) is also drawn as an envelope.

present in the experimental image. The second scenario (shown in the second column) corresponds to intended beam direction at $+4^{\circ}$ on the azimuthal and $0^{\circ}$ on the elevation plane $\left(+4^{\circ}, 0^{\circ}\right)$. A grating lobe is now present at $\left(-6.7^{\circ}, 0^{\circ}\right)$, as expected. The third scenario (shown in the third column) corresponds to intended beam direction at $0^{\circ}$ on the azimuthal and $+4^{\circ}$ on the elevation plane $\left(0^{\circ},+4^{\circ}\right)$. The previous grating lobe is not there anymore, but a new one at $\left(0^{\circ},-5.9^{\circ}\right)$ is now present, close to its expected position. Finally, the fourth scenario (shown in the last column) corresponds to intended beam direction at $+4^{\circ}$ both on the azimuthal and the elevation plane $\left(+4^{\circ},+4^{\circ}\right)$. In this case, grating lobes are present both in the lateral and vertical direction at azimuthal and elevation angles that are practically equal to the expected ones. As it is evident from Fig. 14, the experimental and the simulation images are remarkably similar. This similarity validates our $2 \mathrm{D}$ beam scanning concept, and demonstrates the quality of the fabricated 3D PolyBoard PICs.

Fig. 15 presents an additional compendium of experimental images that validate further our concept and give additional information about the presence of grating lobes in the radiation patterns of the fabricated OPAs. More specifically, Fig. 15 presents two subgroups of images. The first subgroup on the top is associated with the testing of the $2 \times 4$ OPA with $8 \mu \mathrm{m}$ lateral pitch, while the second one in the bottom with the testing of the $2 \times 4$ OPA with $6 \mu \mathrm{m}$ lateral pitch. Each subgroup includes 9 images that correspond to intended beam directions at $-4^{\circ}, 0^{\circ}$ and $+4^{\circ}$ on the azimuthal and the elevation planes. Based on the specific curves in Fig. 7a for 2 AEs with $7.2 \mu \mathrm{m}$ pitch and for 4 AEs with $6 \mu \mathrm{m}$ pitch, the expected spacing between the main and the grating lobes in the images of the second subgroup is

approximately $9.9^{\circ}$ in the vertical and $14.0^{\circ}$ in the lateral corresponds to intended beam direction at $0^{\circ}$ both on the azimuthal and the elevation plane $\left(0^{\circ}, 0^{\circ}\right)$. No grating lobes are
Fig. 15. Experimentally captured images (radiation patterns) of the $2 \times 4$ OPAs (upper subgroup) and $6 \mu \mathrm{m}$ (lower subgroup) lateral pitch. each subgroup, nine beam steering scenarios are presented corresponding

structure. Based on the curves in Fig. 7a for 2 AEs with $7.2 \mu \mathrm{m}$ pitch, and for 4 AEs with $8 \mu \mathrm{m}$ pitch, the expected spacing between the main and the grating lobes in the radiation pattern of this OPA is approximately $9.9^{\circ}$ in the vertical direction and $10.7^{\circ}$ in the lateral direction. Four beam steering scenarios are $10.7^{\circ}$ in the lateral direction. Four beam steering scenarios are 
direction. In both subgroups, the symmetry of the images with respect to the lateral and the vertical axis is evident in the case of symmetrical beam steering directions, which manifests the high performance quality and operation predictability of both OPAs. The spacing between the main and the grating lobes in the vertical direction is approximately $9.9^{\circ}$ in all the images of the first and the third row of both subgroups. This result is expected since the OPAs have the same number of AEs (i.e. 2) and the same pitch $(7.2 \mu \mathrm{m})$ in the vertical direction. In the lateral direction on the other hand, we can observe a significant difference due to the different pitch between the two OPAs. While in the images of the first and the third column of the first subgroup $(8 \mu \mathrm{m}$ pitch) the spacing between the main and the grating lobes is $10.7^{\circ}$, in the corresponding images of the second subgroup ( $6 \mu \mathrm{m}$ pitch) no grating lobes are present. The reason is that the grating lobes in this second subgroup are actually expected to have a spacing of $14^{\circ}$ from the main lobe, and thus to appear at $\pm 10^{\circ}$ in the lateral direction, where they are strongly suppressed by the envelope of the single waveguide emitter.

Fig. 16 presents data from a more in-depth analysis of experimental images. More specifically, Fig. 16a presents the radiation intensity of the $2 \times 4$ OPA with $8 \mu \mathrm{m}$ pitch, when the beam is scanned on the azimuthal plane from $-6^{\circ}$ to $+6^{\circ}$ with $2^{\circ}$ angle step, and its elevation angle is kept $0^{\circ}$. The information associated with the images of the second row in the first subgroup of Fig. 15 is thus included in Fig. 16a. The distribution of the radiation intensity is shown for the slice of $0^{\circ}$ elevation angle, as a function of the azimuthal angle. In a similar way, Fig. $16 \mathrm{~b}$ presents the radiation intensity of the same OPA, when the beam is scanned on the elevation plane from $-4^{\circ}$ to $+4^{\circ}$ with $2^{\circ}$ angle step, and its azimuthal angle is kept $0^{\circ}$. This time, the information associated with the images of the second column in the first subgroup of Fig. 15 is included in Fig. 16b. The distribution of the radiation intensity is shown for the slice of $0^{\circ}$ azimuthal angle, as a function of the elevation angle. Finally, Figs. 16c and 16d present the same information as Figs. 16a and 16b, but for the radiation intensity of the $2 \times 4$ OPA with $6 \mu \mathrm{m}$ pitch. In all these figures, the theoretical radiation intensity of the single end-fire waveguide has been also drawn to make obvious that its distribution serves as an envelope that suppresses the OPA lobes at large angles. As observed, the matching between the theoretical envelopes and the experimental data is satisfactory in all cases, proving again the consistency of the results. The angular spacings between the main lobes and their companion grating lobes are also in good agreement with the theoretical values, as these can be extracted from Fig. 7a, as already described. Finally, more careful inspection of the relevant intensity levels between the main lobes and their companion grating lobes in Figs. 16a to $16 \mathrm{~d}$ reveals that the experimental data are also aligned with the expected values from Figs. $7 \mathrm{~b}$ to $7 \mathrm{~d}$. As example, in the case of an OPA with 4 AEs and $6 \mu \mathrm{m}$ pitch, the theoretical $3 \mathrm{~dB}$ clearance from Fig. $7 \mathrm{~b}$ is $10.8^{\circ}$. The curves in Fig. $16 \mathrm{c}$ for beam direction at $\pm 4^{\circ}$ and $\pm 6^{\circ}$ reveal that the experimental $3 \mathrm{~dB}$ clearance on the azimuthal plane is much larger than $8^{\circ}$, but still smaller than $12^{\circ}$. Use of simple fitting calculations indicate that the actual $3 \mathrm{~dB}$ clearance is in fact close to the theoretical one. The same conclusion about the agreement of the experimental and the theoretical data with respect to the angular clearance can be drawn by similar inspection of other curves in Fig. 16.

\section{CONCLUSIONS AND OUTLOOK}

We have proposed, investigated and experimentally demonstrated a new solution for 2D OPAs in support of laser beam scanning applications. Our solution is based on the use of the 3D PolyBoard platform and the development of PICs with multiple waveguiding layers. The edges of the waveguides at the end-facet of those PICs serve as the individual AEs of the OPAs. The split of the input light among the AEs is realized with the help of lateral and vertical MMI couplers, while the phase of each AE is controlled by a thermal phase shifter with millisecond re-configuration times. Using the field equivalence principle, we have estimated the far-field radiation of the standard PolyBoard waveguide at $1550 \mathrm{~nm}$, and we have calculated that the lobe of the corresponding radiation pattern has a FWHM of $12.7^{\circ}$. We have also investigated the emerging radiation patterns of the linear and plane OPAs in PolyBoard PICs, and we have assessed the impact of the waveguide pitch on the creation of grating lobes and the deterioration of the FOV. Using BPM simulations we have investigated the optical crosstalk between the PolyBoard waveguides as a function of their spacing, and we have concluded that any value down to 6 $\mu \mathrm{m}$ can be considered and used as a safe limit for the prevention of the detrimental coupling between the AEs of the OPAs. When we have at least 4 AEs in a linear array, this cutoff pitch corresponds to an angular spacing of $14.0^{\circ}$ between the main lobe and its adjacent grating lobes, and to an angular clearance of $10.8^{\circ}$, wherein the main lobe of the radiation is higher than any side or grating lobe by at least $3 \mathrm{~dB}$. Based on an extensive set of simulation results, we have designed and fabricated single- and 2-layer PICs on the PolyBoard platform with $1 \times 4$ and $2 \times 4$ OPAs, respectively. The lateral pitch in the OPAs was between 6 and $10 \mu \mathrm{m}$, while the vertical pitch in the $2 \times 4$ OPAs was $7.2 \mu \mathrm{m}$. Using a carefully designed experimental setup, we have demonstrated the potential of the 2-layer PICs to efficiently facilitate 2D beam scanning, and we have validated our simulation results regarding the beam width, the maximum scanning angle and the FOV of the 2D OPAs.

Next steps in our work include the extension of our uniform OPA designs to larger size, starting from a $4 \times 8$ and a $4 \times 16$ OPA in 4-layer PolyBoard PICs. They also include the design of nonuniform 2D OPAs with aperiodic positioning of the AEs in multiples of the operating wavelength $\lambda[44]-[46]$. Such a sparse and aperiodic positioning can result in array factors (AFs) with grating lobes far away from the main beam, and they can thus extend the FOV of the OPAs. Finally, our next steps include the hybrid integration of the PolyBoard PICs with silicon nitride PICs built on TriPleX platform [47]. The aim of this integration is to replace the slow thermal phase shifters on the PolyBoard platform with Lead zirconate titanate (PZT) stress-optic phase shifters on the TriPleX platform since the latter type of phase shifters can potentially offer steering reconfiguration times below $100 \mathrm{~ns}$.

\section{ACKNOWLEDGEMENT}

The present work was supported by the European Commission via the project H2020-ICT-3PEAT (Contract no. 780502). 


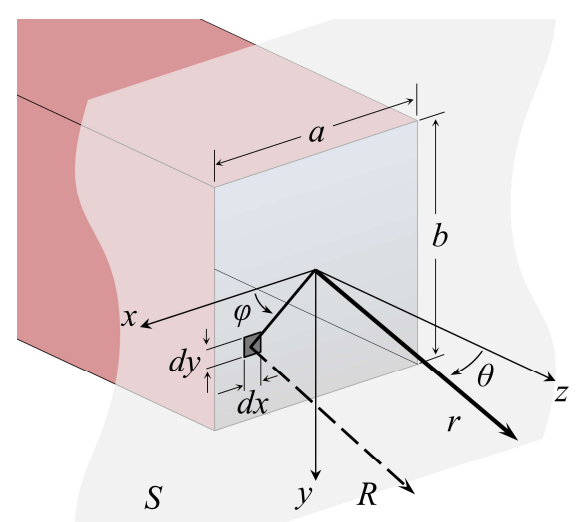

Fig. 17. Extension of Fig. 4a: Coordinate system and geometry for the modelling of the end-fire PolyBoard waveguide as an aperture antenna using the Field Equivalence Principle. The imaginary surface $\mathrm{S}$ that extends to the infinity and encloses the radiation source is also illustrated.

\section{APPENDIX:}

\section{CALCULATION OF THE FAR-FIELD OF AN APERTURE ANTENNA} USING THE FIELD EQUIVALENCE PRINCIPLE

The use of the Field Equivalence Principle for the calculation of the far-field radiation of a rectangular aperture antenna is detailed in [35]. Using the geometry of Fig. 4a, which is presented again in Fig. 17, we can define the plane at the endfacet of the waveguide as an imaginary surface $S$ that extends to the infinity and encloses the actual radiation source. The Field Equivalence Principle allows for replacing this source by equivalent sources with electric current density $\boldsymbol{J}_{S}$ and magnetic current density $\boldsymbol{M}_{S}$. The equivalent sources reside on the surface $S$ and yield the same fields $\boldsymbol{E}_{o}$ and $\boldsymbol{H}_{o}$ as the actual radiation source in the volume outside the surface, which is in fact the volume of interest. The corresponding fields within the volume that is enclosed by the surface can be assumed to take any value. It is obviously convenient to assume that they are zero. In this way, the boundary conditions for the tangential field components on the surface $S$ can be written as:

$\boldsymbol{J}_{S}=\hat{n} \times \boldsymbol{H}_{o}=\hat{z} \times\left(\hat{x} H_{o x}+\hat{y} H_{o y}\right)=\hat{y} H_{o x}-\hat{x} H_{o y}$

$\boldsymbol{M}_{s}=-\hat{n} \times \boldsymbol{E}_{o}=-\hat{z} \times\left(\hat{x} E_{o x}+\hat{y} E_{o y}\right)=-\hat{y} E_{o x}+\hat{x} E_{o y}$

where $\hat{n}$ the unit vector normal to $S$. The components $E_{o x}, E_{o y}$, $H_{o x}$ and $H_{o y}$ at the outer side of the surface can be connected in a straightforward way to the components $E_{x}, E_{y}, H_{x}$ and $H_{y}$ of the waveguided mode taking into account that the physical medium at the outer side of the surface is not the dielectric material of the optical waveguide but the air. As the TE mode has non-zero only the $E_{x}$ and $H_{y}$ components and the TM mode only the $E_{y}$ and $H_{x}$ components, Eq. (4) and (5) can be even simpler if the propagating mode inside the optical waveguide has only TE or TM polarization:

$$
\begin{aligned}
\boldsymbol{J}_{S}^{T E} & =-\hat{x} H_{o y} \\
\boldsymbol{J}_{S}^{T M} & =+\hat{y} H_{o x} \\
\boldsymbol{M}_{S}^{T E} & =-\hat{y} E_{o x} \\
\boldsymbol{M}_{S}^{T M} & =+\hat{x} E_{o y}
\end{aligned}
$$

In either polarization case, the equivalent sources $\boldsymbol{J}_{S}$ and $\boldsymbol{M}_{S}$ derived from Eq. (6)-(9) can be used for the calculation of the normalized magnetic and electric vector potentials $\boldsymbol{N}$ and $\boldsymbol{L}$ related to the far-field radiation. Using again the geometry of Fig. 17, and working with spherical coordinates, the relations between the equivalent sources and the $\hat{\theta}$ and $\hat{\varphi}$ components of the vector potentials take the following form:

$$
\begin{aligned}
& \begin{array}{c}
N_{\theta}(\theta, \varphi)=\iint_{S}\left[J_{x} \cdot \cos \theta \cdot \cos \varphi+J_{y} \cdot \cos \theta \cdot \sin \varphi\right] \\
\cdot e^{i \cdot k \cdot r^{\prime} \cdot \cos \psi} \cdot d s^{\prime}
\end{array} \\
& N_{\varphi}(\theta, \varphi)=\iint_{S}\left[-J_{x} \cdot \sin \varphi+J_{y} \cdot \cos \varphi\right] \cdot e^{i \cdot k \cdot r^{\prime} \cdot \cos \psi} \cdot d s^{\prime} \\
& \begin{array}{c}
L_{\theta}(\theta, \varphi)=\iint_{S}\left[M_{x} \cdot \cos \theta \cdot \cos \varphi+M_{y} \cdot \cos \theta \cdot \sin \varphi\right] \\
\cdot e^{i \cdot k \cdot r^{\prime} \cdot \cos \psi} \cdot d s^{\prime}
\end{array}
\end{aligned}
$$

$L_{\varphi}(\theta, \varphi)=\iint_{S}\left[-M_{x} \cdot \sin \varphi+M_{y} \cdot \cos \varphi\right] \cdot e^{i \cdot k \cdot r^{\prime} \cdot \cos \psi} \cdot d s^{\prime}$

In Eq. (10)-(13), the parameters $\theta$ and $\varphi$ are the spherical coordinates of the observation point in the far-field, $k$ is the wavenumber in free space, $d s^{\prime}=d x^{\prime} \cdot d y^{\prime}$ is the differential area on the surface $S$ of the integration, $r^{\prime}$ is the amplitude of the vector corresponding to the differential area, and $\psi$ the angle between the vectors $\boldsymbol{r}^{\prime}$ and $\boldsymbol{r}$ that correspond to the differential area and the observation point, respectively. For the geometry of Fig. 17, the factor $r^{\prime} \cdot \cos \psi$ that can be seen in all the exponentials of Eq. (10)-(13) takes the following form:

$$
r^{\prime} \cos \psi=x^{\prime} \cdot \sin \theta \cdot \cos \varphi+y^{\prime} \cdot \sin \theta \cdot \sin \varphi
$$

Finally, the electric and magnetic fields that are radiated by the rectangular aperture antenna in the far-field can be calculated using the vector potentials from Eq. (10)-(13) and the following relations:

$$
\begin{gathered}
E_{r}(\theta, \varphi) \approx 0 \\
E_{\theta}(\theta, \varphi) \approx-\frac{i \cdot k \cdot e^{-i \cdot k \cdot r}}{4 \pi r}\left[L_{\varphi}(\theta, \varphi)+\eta \cdot N_{\theta}(\theta, \varphi)\right] \\
E_{\varphi}(\theta, \varphi) \approx \frac{i \cdot k \cdot e^{-i \cdot k \cdot r}}{4 \pi r}\left[L_{\theta}(\theta, \varphi)-\eta \cdot N_{\varphi}(\theta, \varphi)\right] \\
H_{r}(\theta, \varphi) \approx 0
\end{gathered}
$$

$$
H_{\theta}(\theta, \varphi) \approx \frac{i \cdot k \cdot e^{-i \cdot k \cdot r}}{4 \pi r}\left[N_{\varphi}(\theta, \varphi)-\frac{L_{\theta}(\theta, \varphi)}{\eta}\right]
$$




$$
E_{\varphi}(\theta, \varphi) \approx-\frac{i \cdot k \cdot e^{-i \cdot k \cdot r}}{4 \pi r}\left[N_{\theta}(\theta, \varphi)+\frac{L_{\varphi}(\theta, \varphi)}{\eta}\right]
$$

The calculation of the $\boldsymbol{E}$ and $\boldsymbol{H}$ fields from Eq. (15)-(20) can be used in turn for the estimation of other important antenna parameters such as the Poynting vector, the radiation density, the radiation intensity and the beam width [35]. In more detail, the time average Poynting vector is calculated as follows:

$$
\boldsymbol{W}_{a v}(r, \theta, \varphi)=\frac{1}{2} \operatorname{Re}\left[\boldsymbol{E} \times \boldsymbol{H}^{*}\right]
$$

Taking into account that the radial components of the $\boldsymbol{E}$ and $\boldsymbol{H}$ fields are practically zero, Eq. (18) can be written as:

$$
\boldsymbol{W}_{a v}(r, \theta, \varphi)=\frac{1}{2} \operatorname{Re}\left[E_{\theta} \cdot H_{\varphi}^{*}-E_{\varphi} \cdot H_{\theta}^{*}\right] \cdot \hat{r}
$$

The magnitude of the time average Poynting vector in the farfield is denoted as $W_{\text {rad }}$ and describes the radiated power density (or radiation density) in units $\mathrm{W} / \mathrm{m}^{2}$. Finally, the radiation intensity $U_{o}$ is also a far-field parameter that unveils the power radiated by an antenna per unit solid angle. It is expressed in units W/unit solid angle, and is associated with the radiation density via the following mathematical relation:

$$
U_{o}(r, \theta, \varphi)=r^{2} \cdot W_{\text {rad }}(r, \theta, \varphi)
$$

The radiation intensity $U_{o}$ describes in the most intuitive way the radiation pattern and forms the basis for the mathematical calculation of the beamwidth and the directivity of an antenna.

\section{REFERENCES}

[1] X. Sun, L. Zhang, Q. Zhang, and W. Zhang, "Si Photonics for Practical LiDAR Solutions," Applied Sciences, vol. 9, no. 20, p. 4225, Oct. 2019.

[2] Christopher V. Poulton, Ami Yaacobi, David B. Cole, Matthew J. Byrd, Manan Raval, Diedrik Vermeulen, and Michael R. Watts, "Coherent solid-state LIDAR with silicon photonic optical phased arrays," Opt. Lett. 42, 4091-4094 (2017)

[3] C. V. Poulton et al., "Long-Range LiDAR and Free-Space Data Communication With High-Performance Optical Phased Arrays," in IEEE Journal of Selected Topics in Quantum Electronics, vol. 25, no. 5, pp. 1-8, Sept.-Oct. 2019, Art no. 7700108, doi: 10.1109/JSTQE.2019.2908555.

[4] S. Chung, H. Abediasl and H. Hashemi, "A Monolithically Integrated Large-Scale Optical Phased Array in Silicon-on-Insulator CMOS," in IEEE Journal of Solid-State Circuits, vol. 53, no. 1, pp. 275-296, Jan. 2018, doi: 10.1109/JSSC.2017.2757009

[5] Fu, X. J., Yang, F., Liu, C. X., Wu, X. J., Cui, T. J., Terahertz Beam Steering Technologies: From Phased Arrays to Field-Programmable Metasurfaces. Adv. Optical Mater. 2020, 8, 1900628. https://doi.org/10.1002/adom.201900628

[6] Heck, Martijn J.R.. "Highly integrated optical phased arrays: photonic integrated circuits for optical beam shaping and beam steering" Nanophotonics, vol. 6, no. 1, 2017, pp. 93-107. https://doi.org/10.1515/nanoph-2015-0152

[7] K. Van Acoleyen, W. Bogaerts, J. Jágerská, N. Le Thomas, R. Houdré, and R. Baets, "Off-chip beam steering with a one-dimensional optical phased array on silicon-on-insulator," Opt. Lett. 34, 1477-1479 (2009).

[8] K. Van Acoleyen, K. Komorowska, W. Bogaerts and R. Baets, "OneDimensional Off-Chip Beam Steering and Shaping Using Optical Phased Arrays on Silicon-on-Insulator," in Journal of Lightwave Technology, vol. 29, no. 23, pp. 3500-3505, Dec.1, 2011, doi: 10.1109/JLT.2011.2171477.

[9] J. K. Doylend, M. J. R. Heck, J. T. Bovington, J. D. Peters, M. L. Davenport, L. A. Coldren, and J. E. Bowers, "Hybrid III/V silicon photonic source with integrated 1D free-space beam steering," Opt. Lett. $37,4257-4259$ (2012).
[10] Ami Yaacobi, Jie Sun, Michele Moresco, Gerald Leake, Douglas Coolbaugh, and Michael R. Watts, "Integrated phased array for wideangle beam steering," Opt. Lett. 39, 4575-4578 (2014).

[11] J. K. Doylend, M. J. R. Heck, J. T. Bovington, J. D. Peters, L. A. Coldren, and J. E. Bowers, "Two-dimensional free-space beam steering with an optical phased array on silicon-on-insulator," Opt. Express 19, 2159521604 (2011)

[12] David Kwong, Amir Hosseini, John Covey, Yang Zhang, Xiaochuan Xu, Harish Subbaraman, and Ray T. Chen, "On-chip silicon optical phased array for two-dimensional beam steering," Opt. Lett. 39, 941-944 (2014).

[13] J. C. Hulme, J. K. Doylend, M. J. R. Heck, J. D. Peters, M. L. Davenport, J. T. Bovington, L. A. Coldren, and J. E. Bowers, "Fully integrated hybrid silicon two dimensional beam scanner," Opt. Express 23, 58615874 (2015).

[14] David N. Hutchison, Jie Sun, Jonathan K. Doylend, Ranjeet Kumar, John Heck, Woosung Kim, Christopher T. Phare, Avi Feshali, and Haisheng Rong, "High-resolution aliasing-free optical beam steering," Optica 3, 887-890 (2016)

[15] C. V. Poulton, A. Yaacobi, Z. Su, M. J. Byrd, and M. R. Watts, "Optical Phased Array with Small Spot Size, High Steering Range and Grouped Cascaded Phase Shifters," in Advanced Photonics 2016 (IPR, NOMA, Sensors, Networks, SPPCom, SOF), OSA technical Digest (online) (Optical Society of America, 2016), paper IW1B.2.

[16] Steven A. Miller, You-Chia Chang, Christopher T. Phare, Min Chul Shin, Moshe Zadka, Samantha P. Roberts, Brian Stern, Xingchen Ji, Aseema Mohanty, Oscar A. Jimenez Gordillo, Utsav D. Dave, and Michal Lipson, "Large-scale optical phased array using a low-power multi-pass silicon photonic platform," Optica 7, 3-6 (2020).

[17] Yu Zhang, Yi-Chun Ling, Kaiqi Zhang, Cale Gentry, David Sadighi, Greg Whaley, James Colosimo, Paul Suni, and S. J. Ben Yoo, "Subwavelength-pitch silicon-photonic optical phased array for large fieldof-regard coherent optical beam steering," Opt. Express 27, 1929-1940 (2019).

[18] Karel Van Acoleyen, Hendrik Rogier, and Roel Baets, "Twodimensional optical phased array antenna on silicon-on-insulator," Opt. Express 18, 13655-13660 (2010).

[19] Firooz Aflatouni, Behrooz Abiri, Angad Rekhi, and Ali Hajimiri, "Nanophotonic projection system," Opt. Express 23, 21012-21022 (2015).

[20] Sun, J., Timurdogan, E., Yaacobi, A. et al. Large-scale nanophotonic phased array. Nature 493, 195-199 (2013). https://doi.org/10.1038/nature11727

[21] Jie Sun, Ehsan shah Hosseini, Ami Yaacobi, David B. Cole, Gerald Leake, Douglas Coolbaugh, and Michael R. Watts, "Two-dimensional apodized silicon photonic phased arrays," Opt. Lett. 39, 367-370 (2014).

[22] Hooman Abediasl and Hossein Hashemi, "Monolithic optical phasedarray transceiver in a standard SOI CMOS process," Opt. Express 23, 6509-6519 (2015)

[23] C. Qin, K. Shang, S. Feng, G. Liu, S. Pathak and S. J. B. Yoo, "1×256 multi-layer, low-loss, Si3N4 waveguide optical phased arrays with $0.050^{\circ}$ instantaneous-field-of-view," 2017 Conference on Lasers and Electro-Optics (CLEO), San Jose, CA, USA, 2017, pp. 1-2.

[24] Min Chul Shin, Aseema Mohanty, Kyle Watson, Gaurang R. Bhatt, Christopher T. Phare, Steven A. Miller, Moshe Zadka, Brian S. Lee, Xingchen Ji, Ipshita Datta, and Michal Lipson, "Chip-scale blue light phased array," Opt. Lett. 45, 1934-1937 (2020).

[25] M. R. Kossey, C. Rizk, and A. C. Foster, "End-fire silicon optical phased array with half-wavelength spacing," APL Photonics 3(1), 011301 (2018).

[26] C. T. Phare, C. S. Min, S. A. Miller, B. Stern, and M. Lipson, "Silicon Optical Phased Array with High-Efficiency Beam Formation over 180 Degree Field of View," 2018, arXiv:1802.04624 [physics.app-ph].

[27] A. Hosseini, D. Kwong, Y. Zhang, S.A. Chandorkar, F. Crnogorac, A. Carlson, B. Fallah, S. Bank, E. Tutuc, J. Rogers, R.F.W. Pease, R.T. Chen, "On the fabrication of three-dimensional silicon-on-insulator based optical phased array for agile and large angle laser beam steering systems," Journal of Vacuum Science \& Technology B, Nanotechnology and Microelectronics: Materials, Processing, Measurement, and Phenomena, 28(6), C6O1-C6O7 (2010).

[28] Yoo, S., Guan, B. \& Scott, R. Heterogeneous 2D/3D photonic integrated $\begin{array}{lllll}\text { microsystems. Microsyst Nanoeng 2, } 16030 & \text { (2016). }\end{array}$ https://doi.org/10.1038/micronano.2016.30

[29] B. Guan, C. Qin, R. P. Scott, B. Ercan, N. K. Fontaine, T. Su, and S. J. B. Yoo, "Hybrid 3D Photonic Integrated Circuit for Optical Phased 
Array Beam Steering," in CLEO: 2015, OSA Technical Digest (online) (Optical Society of America, 2015), paper STu2F.1.

[30] Gross, S. and Withford, M. J.. "Ultrafast-laser-inscribed 3D integrated photonics: challenges and emerging applications" Nanophotonics, vol. 4, no. 3, 2015, pp. 332-352. https://doi.org/10.1515/nanoph-2015-0020

[31] Zhang, Z.; Felipe, D.; Katopodis, V.; Groumas, P.; Kouloumentas, C.; Avramopoulos, H.; Dupuy, J.-Y.; Konczykowska, A.; Dede, A.; Beretta, A.; Vannucci, A.; Cangini, G.; Dinu, R.; Schmidt, D.; Moehrle, M.; Runge, P.; Choi, J.-H.; Bach, H.-G.; Grote, N.; Keil, N.; Schell, M. Hybrid Photonic Integration on a Polymer Platform. Photonics 2015, 2, 1005-1026. https://doi.org/10.3390/photonics2031005

[32] M. Nuck et al., "Low-Loss Vertical MMI Coupler for 3D Photonic Integration," 2018 European Conference on Optical Communication (ECOC), Rome, Italy, 2018, pp. 1-3, doi: 10.1109/ECOC.2018.8535479.

[33] Z. Zhang et al., "Multicore Polymer Waveguides and Multistep $45^{\circ}$ Mirrors for 3D Photonic Integration," in IEEE Photonics Technology Letters, vol. 26, no. 19, pp. 1986-1989, 1 Oct.1, 2014, doi: 10.1109/LPT.2014.2344116.

[34] David de Felipe, Moritz Kleinert, Crispin Zawadzki, Andrzej Polatynski, Gelani Irmscher, Walter Brinker, Martin Moehrle, Heinz-Gunter Bach, Norbert Keil, and Martin Schell, "Recent Developments in PolymerBased Photonic Components for Disruptive Capacity Upgrade in Data Centers," J. Lightwave Technol. 35, 683-689 (2017)

[35] Antenna Theory: Analysis and Design (3rd Edition) (John Wiley \& Sons, 2005) Author: Constantine A. Balanis.

[36] Kleinert, M., Felipe, D.D., Zawadzki, C., Brinker, W., Choi, J., Reinke, P., Happach, M., Nellen, S., Möhrle, M., Bach, H., Keil, N., \& Schell, M. (2017). Photonic integrated devices and functions on hybrid polymer platform. OPTO

[37] V. Katopodis, P. Groumas, Z. Zhang, R. Dinu, E. Miller, A Konczykowska, J. Y. Dupuy, A. Beretta, A. Dede, J.H. Choi, P. Harati, F. Jorge, V. Nodjiadjim, Muriel Riet, G. Cangini, A. Vannucci, N. Keil, H.-G. Bach, N. Grote, H. Avramopoulos, Ch. Kouloumentas, "Polymer enabled 100Gbaud connectivity for datacom applications," Optics Communications, Volume 362, 2016, Pages 13-21, ISSN 0030-4018, https://doi.org/10.1016/j.optcom.2015.07.064.

[38] P. Groumas et al., "Tunable 100 Gbaud Transmitter Based on Hybrid Polymer-to-Polymer Integration for Flexible Optical Interconnects," in Journal of Lightwave Technology, vol. 34, no. 2, pp. 407-418, 15 Jan. 15, 2016, doi: 10.1109/JLT.2015.2463730.

[39] Madeleine Nuck et al., "3D photonic integrated $4 \times 4$ multi-mode interference coupler," Mar. 2019, vol. 10921, doi: 10.1117/12.2509776.

[40] J. Van Roey, J. van der Donk, and P. E. Lagasse, "Beam-propagation method: analysis and assessment," J. Opt. Soc. Am. 71, 803-810 (1981)

[41] https://www.synopsys.com/photonic-solutions/rsoft-photonic-devicetools/passive-device-beamprop.html

[42] Nicolas Le Thomas, Romuald Houdré, Maria V. Kotlyar, David O'Brien, and Thomas F. Krauss, "Exploring light propagating in photonic crystals with Fourier optics," J. Opt. Soc. Am. B 24, 2964-2971 (2007)

[43] P. Stepanov, A. Delga, N. Gregersen, E. Peinke, M. Munsch, J. Teissier J. Mørk, M. Richard, J. Bleuse, J. M. G'erard, and J. Claudon, "Highly directive and Gaussian far-field emission from "giant" photonic trumpets," Applied Physics Letters 107, 141106 (2015).

[44] D. Kwong, A. Hosseini, Y. Zhang, and R. T. Chen, " $1 \times 12$ Unequally spaced waveguide array for actively tuned optical phased array on a silicon nanomembrane," Appl. Phys. Lett. 99(5), 051104 (2011).

[45] Song, W., Gatdula, R., Abbaslou, S. et al. High-density waveguide superlattices with low crosstalk. Nat Commun 6, 7027 (2015) https://doi.org/10.1038/ncomms 8027

[46] Tin Komljenovic, Roger Helkey, Larry Coldren, and John E. Bowers, "Sparse aperiodic arrays for optical beam forming and LIDAR," Opt. Express 25, 2511-2528 (2017)

[47] http://ict-3peat.eu/ 